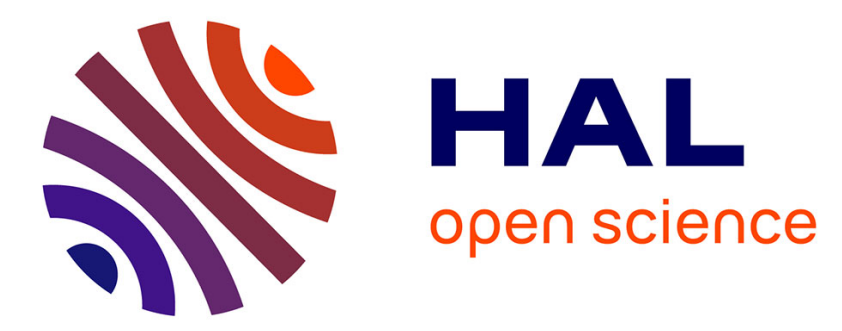

\title{
Experimental study on superharmonic wave generation by resonant interaction between internal wave modes
}

Pauline Husseini, Dheeraj Varma, Thierry Dauxois, Sylvain Joubaud, Philippe Odier, Manikandan Mathur

\section{- To cite this version:}

Pauline Husseini, Dheeraj Varma, Thierry Dauxois, Sylvain Joubaud, Philippe Odier, et al.. Experimental study on superharmonic wave generation by resonant interaction between internal wave modes. Physical Review Fluids, 2020, 5, 10.1103/physrevfluids.5.074804 . hal-02994107

\section{HAL Id: hal-02994107 \\ https://hal.science/hal-02994107}

Submitted on 7 Nov 2020

HAL is a multi-disciplinary open access archive for the deposit and dissemination of scientific research documents, whether they are published or not. The documents may come from teaching and research institutions in France or abroad, or from public or private research centers.
L'archive ouverte pluridisciplinaire HAL, est destinée au dépôt et à la diffusion de documents scientifiques de niveau recherche, publiés ou non, émanant des établissements d'enseignement et de recherche français ou étrangers, des laboratoires publics ou privés. 


\title{
Experimental study on superharmonic wave generation by resonant interaction between internal wave modes
}

\author{
Pauline Husseini, ${ }^{1}$ Dheeraj Varma $\odot,{ }^{1,2}$ Thierry Dauxois $\odot,{ }^{1}$ Sylvain Joubaud $\odot,{ }^{1,3}$ \\ Philippe Odier, ${ }^{1}$ and Manikandan Mathur $\circledast^{1,2}$ \\ ${ }^{1}$ Université de Lyon, ENS de Lyon, Université Claude Bernard, CNRS, Laboratoire de Physique, \\ F-69342 Lyon, France \\ ${ }^{2}$ Department of Aerospace Engineering, Indian Institute of Technology Madras, Chennai 600036, India \\ ${ }^{3}$ Institut Universitaire de France (IUF), F-75005 Paris, France
}

(Received 11 December 2019; accepted 7 July 2020; published 30 July 2020)

\begin{abstract}
We present an experimental study of resonant generation of superharmonic internal waves as a result of interaction between horizontally propagating vertical internal wave modes $m$ and $n$ at frequency $\omega_{0}$ in a uniformly stratified finite-depth fluid. Thorpe [J. Fluid Mech. 24, 737 (1966)] has shown theoretically that modes $m$ and $n$ at frequency $\omega_{0}$ and mode $p=|m-n|$ at frequency $2 \omega_{0}$ are in triadic resonance at specific values of $\omega_{0}$. We demonstrate the occurrence of this triadic resonance by forcing a primary wave field of modes $m$ and $n$ at various $\omega_{0}$ using a novel internal wave generator, and observing the spontaneous growth (or lack thereof) of the superharmonic mode $p=|m-n|$ at frequency $2 \omega_{0}$. A superharmonic wave field with a predominantly mode- $p=|m-n|$ structure is observed over a finite range of frequency $\left(\Delta \omega_{0} \simeq 0.03 N\right)$ around the resonant value, where $N$ is the uniform buoyancy frequency. The spatial growth of the superharmonic wave field is then quantitatively measured, to subsequently compare with the predictions from amplitude evolution equations at resonance at various forcing amplitudes, thereby validating this model. It is furthermore shown that a large-scale spatial evolution of the wave field is more suited to describe our experiments than the slow temporal evolution approach. The paper concludes with a brief discussion of viscous effects.
\end{abstract}

DOI: 10.1103/PhysRevFluids.5.074804

\section{INTRODUCTION}

Internal waves, which are propagating disturbances in stably stratified fluids, have been a subject of active research in the last few decades. In oceanic and atmospheric applications, their potential to transport energy and momentum to large horizontal and vertical distances has important implications [1]. Furthermore, they transfer energy irreversibly from large spatial scales (tides, winds) to significantly smaller scales in the ocean, ultimately contributing to small-scale turbulence and mixing [2]. From a fundamental point of view, the internal wave dispersion relation [3] contains several interesting characteristics: (i) anisotropic propagation, (ii) single frequency supporting an infinite number of wave numbers, and (iii) orthogonality between phase and group velocities for plane internal waves. Hence, the manifestations of classical wave phenomena such as resonant generation $[4,5]$, scattering by topography [6,7] and nonuniform stratifications $[8,9]$, wave-wave interactions $[10,11]$, and solitary wave generation $[12,13]$ represent fascinating topics in internal waves.

In a uniformly stratified fluid of infinite depth, the dispersion relation for plane internal waves in the two-dimensional $x z$ plane is

$$
\sin ^{2} \theta=\frac{\omega^{2}}{N^{2}}
$$


where $\omega$ is the internal wave frequency and $N$ the constant buoyancy frequency determined by the background density gradient. The angle $\theta$ represents the angle between the wave vector $\mathbf{k}$ and the vertical $z$ axis (gravity acts along the negative $z$ axis). Alternately, $\theta$ also represents the angle between the group velocity and the horizontal $x$ axis. Therefore, for given values of $\omega$ and $N$, the horizontal and vertical wave numbers can take any value in the range of $(-\infty,+\infty)$, while being related by Eq. (1). In the presence of horizontal boundaries, however, the vertical wave numbers can get discretized owing to boundary conditions. Specifically, with rigid horizontal boundaries at $z=0$ and $z=H$ where no-normal-flow is enforced, the vertical structure of the stream function is constrained to be of the form $\sin (n \pi z / H)$, where the mode number $n$ takes integer values. As a consequence, the horizontal wave numbers are also discretized owing to the dispersion relation, and are given by $k=n \pi /(H \cot \theta)$, with propagation occurring only along the horizontal. It is also instructive to interpret an individual mode $n$ as a linear superposition of upward and downward propagating plane waves (of the same amplitude) with wave vectors $(k, n \pi / H)$ and $(k,-n \pi / H)$. While plane-wave studies in infinite depth are often insightful, there can also be significant deviations when finite-depth effects are included. For example, internal tide generation in a finite-depth ocean can be substantially weaker than in an infinite-depth ocean if the topographic and internal tide length scales are comparable [14]. In the current study, we investigate resonant and near-resonant wave-wave interactions in internal wave modes.

For oceanic applications, internal wave research has focused broadly on two different questions. The first relates to mechanisms via which internal waves are generated, the amount of energy input into them, and their spatial/temporal scales. Reasonable estimates are now available on internal tide generation by barotropic forcing on bottom topography [15] and wind-forced near-inertial waves in the upper ocean [16]. The second question relates to the mechanisms via which the internal wave energy gets dissipated. Several mechanisms have been proposed, summaries of which can be found in [17-19]. Triadic resonance represents one such pathway towards dissipation, where energy is transferred to other frequencies and wave numbers. Specifically, three plane internal waves that satisfy $\omega_{1}+\omega_{2}+\omega_{3}=0$ (positive or negative frequencies $\omega_{1,2,3}$ ) and $\mathbf{k}_{\mathbf{1}}+\mathbf{k}_{\mathbf{2}}+\mathbf{k}_{\mathbf{3}}=0$ spontaneously exchange energy between each other, and are termed together as a resonant triad. In fact, triadic resonance is the underlying mechanism for almost all instabilities in finite-amplitude plane internal waves [20,21]. Triadic resonance is possible in internal wave modes, too [22], and this paper presents an experimental investigation of the same.

Most studies on triadic resonance consider a primary wave with a finite amount of initial energy and two secondary daughter waves with an infinitesimally small initial energy. Those resonant triads for which the frequencies of the secondary waves are smaller (in magnitude) than the primary wave frequency are referred to as subharmonic resonant triads. For plane internal waves in a uniform stratification, Hasselmann [10] has theoretically shown that subharmonic resonant triads are unstable, i.e., the primary wave would lose its energy to the subharmonic daughter waves. Several numerical studies have then performed detailed investigations of subharmonic triadic resonance in plane waves [23] and realistic ocean settings [24]. In laboratory settings, finite-depth effects are inevitable, and primary standing internal wave modes have been shown to generate other modes at subharmonic frequencies [25-28]. In contrast to subharmonic triadic resonance, it is also possible that the primary wave field contains two waves of a resonant triad, which in turn generates a superharmonic wave whose frequency is the sum of the primary wave frequencies. We term such resonant triads as superharmonic resonant triads, a classic example of which is the generation of superharmonic internal waves by colliding wave beams [29-31]. Numerical studies have shown that subharmonic and superharmonic frequencies are simultaneously generated as a result of wave-wave interactions [32].

One of the challenges in laboratory experiments to study internal wave triadic resonance is to force a desired primary wave field, i.e., input specific amounts of energy into desired frequencies and wave numbers. The internal wave generator described in [33] enables the excitation of various internal wave forms, as demonstrated in [34]. Indeed, experimental studies on subharmonic triadic resonance in a plane internal wave [35] and a mode- 1 internal wave [36] have been performed using 
the internal wave generator. In this paper, we use this generator to force a primary wave field that contains two different modes at the same frequency, and study the superharmonic wave generation due to triadic resonance. The theoretical prediction [22] of the resonant forcing frequency and the superharmonic mode number are verified, the theoretical estimates [37] of energy transfer rates are validated, and near-resonant behavior is investigated.

The rest of the paper is organized as follows. Section II presents the details of our experimental setup and measurement techniques. Section III presents the theory that motivates our experiments and the amplitude evolution equations that are validated by our experiments. Our experimental results are presented in Sec. IV, followed by discussions in Sec. V. A summary of conclusions and future directions are provided in Sec. VI.

\section{EXPERIMENT AND DATA PROCESSING}

As mentioned in Sec. I, we performed experiments to investigate superharmonic resonant triads, where the primary wave field comprises modes $m$ and $n$ at frequency $\omega_{0}$. In a uniform stratification $N$, internal wave modes $m$ and $n(m \neq n)$ at frequency $\omega_{0}$ are in triadic resonance with mode $p=$ $|m-n|$ at frequency $2 \omega_{0}$ if

$$
\frac{\omega_{0}^{2}}{N^{2}}=\frac{(m+n)^{2}-4(m-n)^{2}}{4\left[(m+n)^{2}-(m-n)^{2}\right]},
$$

along with $m / 3<n<3 m$, is satisfied [22]. As described below, the internal wave generator was tuned to excite the aforementioned primary wave field. The growth of the superharmonic wave field was then quantitatively measured, and compared with the predictions from the amplitude evolution equations presented in Sec. III. Experiments were also performed at near-resonant frequencies to measure the corresponding resonance width.

Experiments were performed in an acrylic tank of 4-m length and 17-cm width. The tank was composed of five different components that were each of $0.8 \mathrm{~m}$ in length, joined together using screws in the threaded holes on their frames, with a rubber gasket at the joints (black vertical strips in Fig. 1) to prevent leaks. In every experiment, a uniform stratification with $N=\sqrt{\left(-g / \rho_{\text {ref }}\right) d \rho_{0} / d z} \approx 1 \mathrm{rad} / \mathrm{s}$ over a depth of $H=32.5 \mathrm{~cm}$ was set up using the double-bucket method [38], with the salinity of the water uniquely determining the density $\left(\rho_{0}\right)$ at different depths in the tank. Here, $(x, z)$ denote the horizontal and vertical coordinates, with gravity $g=9.8 \mathrm{~m} / \mathrm{s}^{2}$ acting along negative $z$, and $\rho_{\text {ref }}=1000 \mathrm{~kg} / \mathrm{m}^{3}$ is a reference density. The entire experimental facility was maintained at constant room temperature ensuing the background stratification to be function of salinity alone. After each filling, the density profile in the tank was accurately measured using a conductivity-temperature (CT) probe, which was a priori calibrated using known density samples. In every experiment, a linear fit closely captured the density profile over the entire tank depth except near the boundaries, and was used to estimate the uniform stratification $N$. A sample density profile measured in one of our experiments, along with a linear fit, is shown at the right end of Fig. 1, which also shows a schematic of the entire experimental setup.

Internal waves at a fixed frequency $\omega_{0}$ (time period $T_{0}=2 \pi / \omega_{0}$ ), and comprising specific modes, were forced at the left end of the tank using an internal wave generator whose principle of operation was similar to what is reported in [34]. The internal wave generator comprised 50 plates of 6.5-mm thickness each, and they were independently driven by 50 different motors. More details of this wave generator, which was previously used in [39], can be found in [40]. Each plate executed constant amplitude oscillations along the horizontal such that the vertical profile of the horizontal displacement field at the wave generator location $(x=0)$ was given by ( $t$ denotes time)

$$
\Gamma_{x}(x=0, z, t)=\left[A_{m} \cos \left(\frac{m \pi z}{H}\right)+A_{n} \cos \left(\frac{n \pi z}{H}\right)\right] \cos \omega_{0} t,
$$

thus comprising left-to-right propagating modes $m$ and $n$ at amplitudes $A_{m}$ and $A_{n}$, respectively. Since the wave generator forces only the horizontal velocity, and its forcing is (i) discretized (finite 


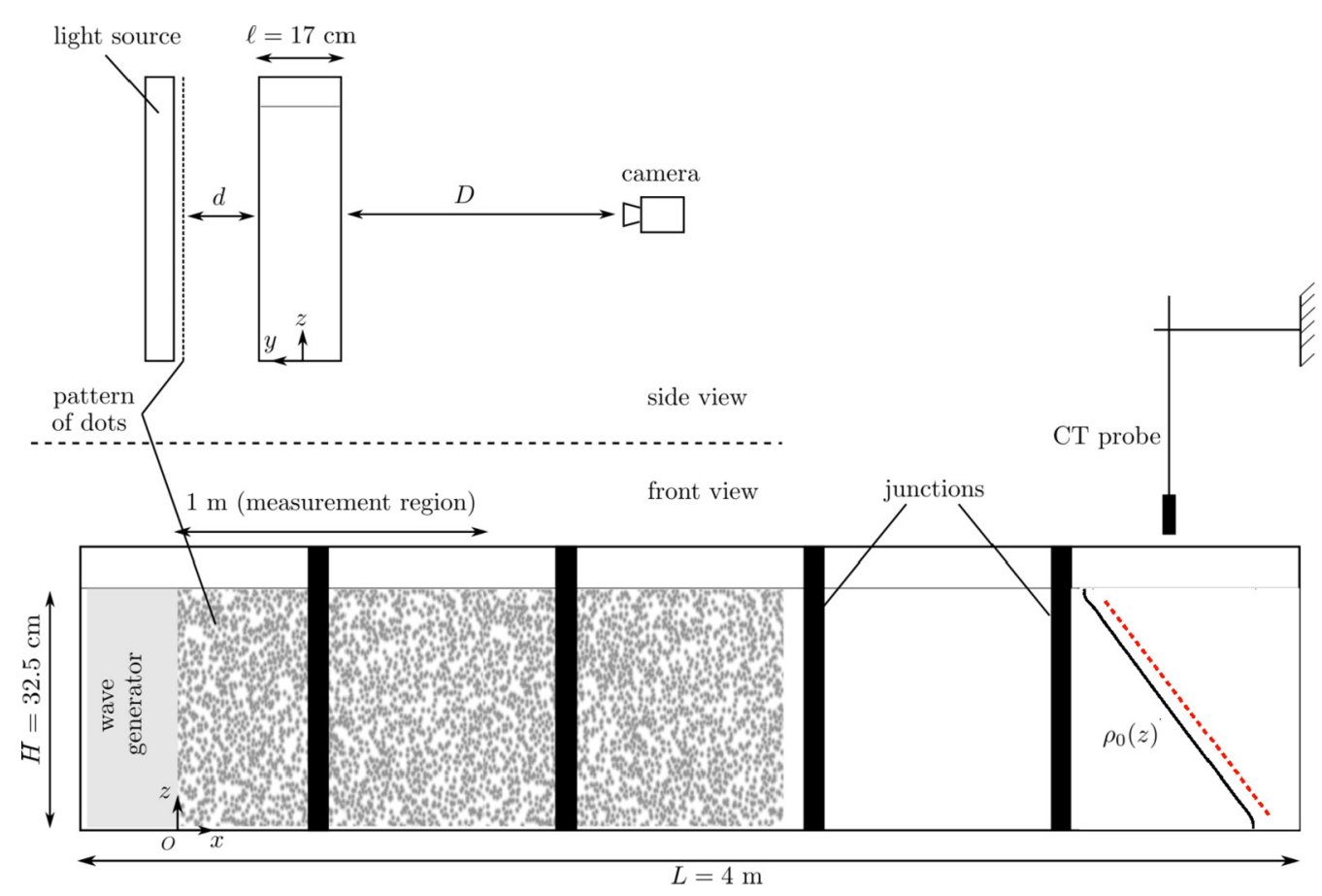

FIG. 1. Schematic of the experimental setup used in the current study. The uniform stratification in the tank was measured using a conductivity-temperature (CT) probe before the wave generator was started. A sample measured density profile (black solid line) and a linear fit (red dashed-dotted line) are shown at the right end of the tank. Typical values of $\rho_{0}(z)$ at the bottom and top of the tank were $1033.1 \mathrm{~kg} / \mathrm{m}^{3}$ and 1000 $\mathrm{kg} / \mathrm{m}^{3}$, respectively. A uniform density gradient estimated by the linear fit (red dashed line) corresponds to $N=1 \mathrm{rad} / \mathrm{s}$.

thickness of the plates) and (ii) not strictly at a fixed $x$ location (moving plates), not all its forcing is converted into internal waves. Hence, the measured modal amplitudes even in a close neighborhood of the wave generator would be weaker than what are enforced according to Eq. (3). We also note that the wave generator is started impulsively in every experiment, which would result in transient features in the beginning of the experiment. The transient features, however, are unlikely to influence the steady-state wave field.

Quantitative measurements of the instantaneous density gradient perturbation fields were performed using the synthetic Schlieren technique [41]. A back-lit random pattern of dots, as shown in Fig. 1, was placed at a distance of $d=12.5 \mathrm{~cm}$ behind the back wall of the tank. A $2452 \times$ 2054 pixels CCD camera (AVT Pike F-505), looking through the tank, captured images (at $5 \mathrm{~Hz}$ ) of the random pattern of dots from a distance of $D=280 \mathrm{~cm}$ from the front wall of the tank. Each of the captured images, along with the reference image captured with no flow, was analyzed to estimate the apparent displacement of the dots caused by the density gradient perturbations in the flow. The apparent displacement field was estimated using correlation image velocimetry [42], implemented in the open source software UVMAT [43]. The final output of the processing of the images was a quantitative measurement (at $5 \mathrm{~Hz}$ ) of the horizontal and vertical density gradient perturbation fields specified on a $128 \times 128$ grid, spanning a physical domain of $1 \mathrm{~m}$ in the horizontal and the water depth of $32.5 \mathrm{~cm}$ in the vertical.

The finite time Fourier transform of any measured quantity $Q(x, z, t)$ is denoted as $\widehat{Q}(x, z, t, \omega)$, which is then used to reconstruct the quantity filtered at any desired frequency $\omega$ (denoted $\left.Q\right|^{\omega}$ ). For the Fourier transform operation, a time window of $5 T_{0}$ centered around a given time $t$ is used 
to obtain $\widehat{Q}$ corresponding to $t$. This relatively short time window had to be chosen so that it was possible to identify a steady-state period before the reflected waves from the right end of the tank reached the measurement domain. For the inverse Fourier transform operation used to obtain $\left.Q\right|^{\omega}$, a frequency window of $0.8 \omega$ to $1.2 \omega$ is used. This relatively large frequency window is required to entirely capture the spectral peaks, whose widths are in turn determined by the time window $\left(5 T_{0}\right)$ we use for calculating the spectra. The measured density gradient perturbation fields, filtered at a frequency $\omega$, are then written as

$$
\begin{aligned}
& \left.\frac{\partial \rho^{\prime}}{\partial x}(x, z, t)\right|^{\omega}=\sum_{q=1}^{\infty} R_{q}^{\omega}(x, t) \sin \left(\frac{q \pi z}{H}\right) \cos \left(k_{q}^{\omega} x-\omega t+\gamma_{q}^{\omega}\right), \\
& \left.\frac{\partial \rho^{\prime}}{\partial z}(x, z, t)\right|^{\omega}=\sum_{q=1}^{\infty} S_{q}^{\omega}(x, t) \cos \left(\frac{q \pi z}{H}\right) \sin \left(k_{q}^{\omega} x-\omega t+\gamma_{q}^{\omega}\right),
\end{aligned}
$$

where $k_{q}^{\omega}=q \pi /\left(H \cot \theta^{\omega}\right)$ with $\cot \theta^{\omega}=\sqrt{N^{2}-\omega^{2}} / \omega$, and $\gamma_{q}^{\omega}$ the associated phase. The vertically integrated horizontal energy flux in mode $q$ at frequency $\omega$, averaged over one time period, is given by

$$
E_{q}^{\omega}=\frac{g^{2} H^{4}}{4 \pi^{3} \rho_{\mathrm{ref}} N q^{3}} \frac{N^{2}}{\omega^{2}}\left(1-\frac{\omega^{2}}{N^{2}}\right)^{5 / 2}\left(R_{q}^{\omega}\right)^{2} .
$$

The spatial evolution of the modal amplitudes $R_{q}^{\omega}(x, t)$ and $S_{q}^{\omega}(x, t)$ [estimated by a modal decomposition of the measured density gradient fields, as in Eqs. (4) and (5)] is then compared with the predictions based on the amplitude evolution equations in Sec. III B. It is noteworthy that comparisons with the theory presented in Sec. III B require steady-state amplitudes from the experiments, which we estimate by waiting sufficiently long for steady state to be reached at a given $x$, but not so long that the reflected waves from the right end of the tank affect our estimates. Typically, experimental measurements during $20 T_{0}$ to $25 T_{0}$ were observed to be reliable estimates of the steady-state wave field. As an estimate of sensitivity to the specific steady-state period used in our analysis, we measured the change in the superharmonic modal amplitude at the center of the measurement domain to be around $2.5 \%$ if the steady-state period is changed to $25 T_{0}-30 T_{0}$. While the initiation of the superharmonic growth occurs rather quickly at all spatial locations in our measurement domain, the time required to reach steady state is different at different $x$. Owing to the difficulty posed by the reflected wave field, we refrained from choosing different steady-state periods for different spatial locations.

Experiments were performed to study two different modal interactions.

Case 1. $(m, n, p)=(3,4,1)$ : mode 3 and mode 4 at $\omega_{0}$, which are in triadic resonance with mode 1 at $2 \omega_{0}$ if $\omega_{0} / N=\sqrt{45 / 192} \approx 0.4841$, based on theory [Eq. (2)]. At resonance, assuming $H=32.5 \mathrm{~cm}$, the theoretical estimates of horizontal wavelengths $\left(\lambda_{i}=2 \pi / k_{i}, i=3,4,1\right)$ of the three waves are $\lambda_{3}=0.392 \mathrm{~m}, \lambda_{4}=0.294 \mathrm{~m}$, and $\lambda_{1}=0.168 \mathrm{~m}$. Based on the group velocity of the respective modes, it would take $25 T_{0}, 33 T_{0}$, and $352 T_{0}$ for primary mode 3 , primary mode 4 , and superharmonic mode 1 to reach $x=0.6 \mathrm{~m}$ after being reflected from the right end of the tank.

Case $2 .(m, n, p)=(3,5,2)$ : mode 3 and mode 5 at $\omega_{0}$, which are in triadic resonance with mode 2 at $2 \omega_{0}$ if $\omega_{0} / N=\sqrt{1 / 5} \approx 0.4472$, based on theory [Eq. (2)]. At resonance, assuming $H=32.5$ $\mathrm{cm}$, the theoretical estimates of horizontal wavelengths $\left(\lambda_{i}=2 \pi / k_{i}, i=3,5,2\right)$ of the three waves are $\lambda_{3}=0.433 \mathrm{~m}, \lambda_{5}=0.26 \mathrm{~m}$, and $\lambda_{2}=0.163 \mathrm{~m}$. Based on the group velocity of the respective modes, it would take $21 T_{0}, 36 T_{0}$, and $113 T_{0}$ for primary mode 3 , primary mode 5 , and superharmonic mode 2 to reach $x=0.6 \mathrm{~m}$ after being reflected from the right end of the tank.

For both cases, experiments were performed for forcing frequencies at and around the resonant frequency so as to demonstrate the occurrence of resonance, investigate the wave field at offresonant frequencies, and validate the theoretical predictions at resonance. Further experiments were performed at resonance for case 1 to study the effect of forcing amplitudes. 


\section{THEORY}

In this section, we present the theoretical ideas that motivate our experiments, which concern the nonlinear effects that result from the interaction between two internal wave modes at the same frequency. The equations governing an inviscid, incompressible, nonrotating, two-dimensional flow within the Boussinesq approximation are

$$
\begin{aligned}
\frac{\partial^{2}}{\partial t^{2}} \nabla^{2} \psi+N^{2} \frac{\partial^{2} \psi}{\partial x^{2}} & =\frac{\partial}{\partial x}[J(\psi, b)]-\frac{\partial}{\partial t}\left[J\left(\psi, \nabla^{2} \psi\right)\right], \\
\frac{\partial b}{\partial t} & =-J(\psi, b)+N^{2} \frac{\partial \psi}{\partial x}
\end{aligned}
$$

where $\psi(x, z, t)$ and $b(x, z, t)$ represent the stream function and buoyancy perturbation, respectively. The background stratification is specified by the buoyancy frequency $N$, which is assumed constant in the rest of this paper. The Jacobian and Laplacian operators are defined as $J(A, B)=$ $(\partial A / \partial x)(\partial B / \partial z)-(\partial B / \partial x)(\partial A / \partial z)$ and $\nabla^{2}=\partial^{2} / \partial x^{2}+\partial^{2} / \partial z^{2}$, respectively. The velocity field is given by $(u, w)=(-\partial \psi / \partial z, \partial \psi / \partial x)$.

We consider a flow field given by a regular perturbation series in a small nonlinearity parameter $\epsilon$,

$$
\begin{gathered}
\psi(x, z, t)=\epsilon \psi_{1}(x, z, t)+\epsilon^{2} \psi_{2}(x, z, t)+O\left(\epsilon^{3}\right)+\ldots . \\
b(x, z, t)=\epsilon b_{1}(x, z, t)+\epsilon^{2} b_{2}(x, z, t)+O\left(\epsilon^{3}\right)+\ldots .
\end{gathered}
$$

representing perturbations to the background flow state described by a quiescent fluid with a constant stable stratification $N$.

\section{A. Constant-amplitude solutions}

Assuming the wave field at $O(\epsilon)$ to be described by a superposition of linear internal wave modes $m$ and $n$ at frequency $\omega_{0}$ in a uniformly stratified fluid of depth $H$, we write

$$
\begin{gathered}
\psi_{1}(x, z, t)=\frac{\Psi_{m}}{2} \sin \left(\frac{m \pi z}{H}\right) e^{i\left(k_{m} x-\omega_{0} t\right)}+\frac{\Psi_{n}}{2} \sin \left(\frac{n \pi z}{H}\right) e^{i\left(k_{n} x-\omega_{0} t\right)}+\text { c.c. } \\
b_{1}(x, z, t)=-N^{2}\left[\frac{\Psi_{m} k_{m}}{2 \omega_{0}} \sin \left(\frac{m \pi z}{H}\right) e^{i\left(k_{m} x-\omega_{0} t\right)}+\frac{\Psi_{n} k_{n}}{2 \omega_{0}} \sin \left(\frac{n \pi z}{H}\right) e^{i\left(k_{n} x-\omega_{0} t\right)}+\text { c.c. }\right],
\end{gathered}
$$

where c.c. denotes complex conjugate. The complex amplitudes $\Psi_{m}$ and $\Psi_{n}$ are assumed to be constant in this subsection. The constant-amplitude assumption leads to the divergence of $\psi_{2}$ if the modes in $\psi_{1}$ are part of a resonant triad, and is hence valid only away from triadic resonance. The horizontal wave numbers $k_{m}$ and $k_{n}$ are given by $k_{m}=m \pi /\left(H \cot \theta_{0}\right)$ and $k_{n}=n \pi /\left(H \cot \theta_{0}\right)$, where $\cot \theta_{0}=\sqrt{N^{2}-\omega_{0}^{2}} / \omega_{0}$ results from the linear internal wave dispersion relation (1). Note that the no-normal-flow boundary condition is satisfied at the horizontal boundaries at $z=0$ and $z=H$.

Substituting Eqs. (9) and (10) in the governing Eqs. (7) and (8), with $\psi_{1}$ and $b_{1}$ being given by Eqs. (11) and (12), the flow field at $O\left(\epsilon^{2}\right)$ can be shown to be governed by a linear PDE forced by terms involving the $O(\epsilon)$ flow field. The particular solution of the resulting PDE is

$$
\begin{aligned}
\psi_{2}(x, z, t) & =I_{m n} \sin \left(\frac{(m-n) \pi z}{H}\right) \frac{\Psi_{m} \Psi_{n}}{4} e^{i\left[\left(k_{m}+k_{n}\right) x-2 \omega_{0} t\right]}, \\
& +J_{m n} \sin \left(\frac{(m+n) \pi z}{H}\right) \frac{\Psi_{m} \Psi_{n}^{*}}{4} e^{i\left[\left(k_{m}-k_{n}\right) x\right]}+\text { c.c. },
\end{aligned}
$$

where

$$
I_{m n}=\frac{3 m n\left(m^{2}-n^{2}\right)}{\cot \theta_{0}} \frac{N^{2}}{\omega_{0}}\left(\frac{\pi}{H}\right)^{2}\left(\frac{1}{(m+n)^{2}\left(N^{2}-4 \omega_{0}^{2}\right)-(m-n)^{2} 4 \omega_{0}^{2} \cot ^{2} \theta_{0}}\right),
$$


and

$$
J_{m n}= \begin{cases}\frac{m n}{\omega_{0} \cot \theta_{0}}\left(\frac{\pi}{H}\right)^{2}, & m \neq n \\ 0, & m=n .\end{cases}
$$

The weakly nonlinear solution (13) thus comprises one term at frequency $2 \omega_{0}$ and another at zero frequency, with the latter corresponding to a Eulerian mean flow. The corresponding buoyancy $b_{2}(x, z, t)$ at $O\left(\epsilon^{2}\right)$ is given by

$$
b_{2}(x, z, t)=a_{m n} \sin \left(\frac{(m-n) \pi z}{H}\right) \frac{\Psi_{m} \Psi_{n}}{4} e^{i\left[\left(k_{m}+k_{n}\right) x-2 \omega_{0} t\right]}+\text { c.c. }
$$

where

$$
a_{m n}=\frac{m n(n-m) N^{2}}{\omega_{0}^{2} \cot ^{2} \theta_{0}}\left(\frac{\pi}{H}\right)^{3}\left(\frac{(m+n)^{2}\left(N^{2}+2 \omega_{0}^{2}\right)+(m-n)^{2} 2 \omega_{0}^{2} \cot ^{2} \theta_{0}}{(m+n)^{2}\left(N^{2}-4 \omega_{0}^{2}\right)-(m-n)^{2} 4 \omega_{0}^{2} \cot ^{2} \theta_{0}}\right),
$$

thus comprising only a term at frequency $2 \omega_{0}$. The amplitude of the term at frequency $2 \omega_{0}$ in Eqs. (13) and (16) diverges if the denominator in Eq. (14) goes to zero, which occurs exactly when Eq. (2) is satisfied. This divergence was previously shown in [22], and extended further to the case of nonzero background rotation and nonuniform stratification in [44]. In other words, for the values of $\omega_{0}$ given by Eq. (2), modes $m$ and $n$ at frequency $\omega_{0}$ are in triadic resonance with mode $|m-n|$ at frequency $2 \omega_{0}$. The divergence of $\psi_{2}$ implies that the constant-amplitude solution forms for $\psi_{1}$ [Eq. (11)] are not valid at triadic resonance.

For the triadic resonance that results from the frequency $\omega_{0}$ satisfying Eq. (2), allowing temporal or spatial evolution of the modal amplitudes could rectify the unphysical divergence of the flow field at $O\left(\epsilon^{2}\right)$. Such amplitude evolution equations are derived using the classical method of multiple scales [45], where it is assumed that the temporal or spatial scales over which the modal amplitudes evolve are much larger than those corresponding to the frequencies or spatial wave numbers of the waves.

\section{B. Amplitude evolution equations}

The constant-amplitude theory presented in Sec. III A, while being useful to identify parameters for which triadic resonance occurs, breaks down at triadic resonance due to the divergence of the flow field at $O\left(\epsilon^{2}\right)$. In this subsection, we present results from a complementary theoretical approach that strictly assumes triadic resonance. In our laboratory experiments, a wave generator continuously forces the primary waves from a spatially local region, i.e., around $x=0$. The wave field then evolves nonlinearly as it propagates away from the wave generator. Thus, we assume the existence of a slowly varying spatial coordinate $X=\epsilon x$ over which the internal wave modal amplitudes vary. At the leading order $O(\epsilon)$, the wave field comprises all three waves forming the resonant triad, i.e., modes $m$ and $n$ at frequency $\omega_{0}$ and mode $p=|m-n|$ at frequency $2 \omega_{0}$, given as

$$
\begin{gathered}
\psi_{1}(x, z, t)=\frac{\Psi_{m}(X)}{2} \sin \left(\frac{m \pi z}{H}\right) e^{i\left(k_{m} x-\omega_{0} t\right)}+\frac{\Psi_{n}(X)}{2} \sin \left(\frac{n \pi z}{H}\right) e^{i\left(k_{n} x-\omega_{0} t\right)} \\
+\frac{\Psi_{p}(X)}{2} \sin \left(\frac{(n-m) \pi z}{H}\right) e^{i\left(k_{p} x-2 \omega_{0} t\right)}+\text { c.c. } \\
b_{1}(x, z, t)=-N^{2}\left[\frac{\Psi_{m}(X) k_{m}}{2 \omega_{0}} \sin \left(\frac{m \pi z}{H}\right) e^{i\left(k_{m} x-\omega_{0} t\right)}+\frac{\Psi_{n}(X) k_{n}}{2 \omega_{0}} \sin \left(\frac{n \pi z}{H}\right) e^{i\left(k_{n} x-\omega_{0} t\right)}\right. \\
\left.+\frac{\Psi_{p}(X) k_{p}}{4 \omega_{0}} \sin \left(\frac{(n-m) \pi z}{H}\right) e^{i\left(k_{p} x-2 \omega_{0} t\right)}+\text { c.c. }\right] .
\end{gathered}
$$

Here, the horizontal wave number $k_{p}=p \pi /\left(H \cot \theta_{p}\right)$, with $\cot \theta_{p}=\sqrt{N^{2}-4 \omega_{0}^{2}} /\left(2 \omega_{0}\right)$, satisfies $k_{p}=k_{m}+k_{n}$, thus causing triadic resonance. In terms of the stream-function amplitudes, the modal 
(a)

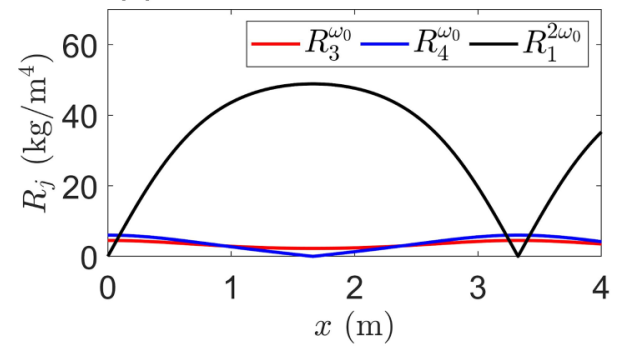

(b)

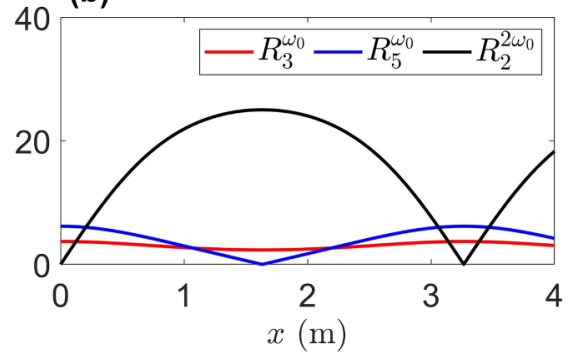

FIG. 2. Theoretical spatial evolution of the modal amplitudes $R_{j}$ of $\partial \rho^{\prime} / \partial x$ for the (a) case-1 resonant triad, i.e., $(m, n, p)=(3,4,1)$ at $\omega_{0} / N=0.4841$ and (b) case-2 resonant triad, i.e., $(m, n, p)=(3,5,2)$ at $\omega_{0} / N=0.4472$. In each of the plots, the amplitudes of mode $m$ at $\omega_{0}$, mode $n$ at $\omega_{0}$, and mode $p$ at $2 \omega_{0}$ are shown in red, blue, and black, respectively. The plots in this figure have been obtained by numerically solving Eqs. (20) and (21) for the corresponding resonant triad, with $H=0.325 \mathrm{~m}$ and $N=1 \mathrm{rad} / \mathrm{s}$. The initial conditions $\Psi_{m}(X=0)$ and $\Psi_{n}(X=0)$ correspond to $\left(A_{m}, A_{n}\right)=(5,5) \mathrm{mm}$ in Eq. (3), with $\Psi_{p}(X=0)=0$.

amplitudes of the horizontal displacement forced by the wave generator $A_{m}$ and $A_{n}$ in Eq. (3) can be written as $\left|A_{m}\right|=\epsilon\left|\Psi_{m}(X=0)\right| m \pi /\left(H \omega_{0}\right)$ and $\left|A_{n}\right|=\epsilon\left|\Psi_{n}(X=0)\right| n \pi /\left(H \omega_{0}\right)$, where $\epsilon$ appears since $\epsilon \psi_{1}$ is what represents the primary wave field.

The amplitude evolution equations can be shown to be [37]

$$
\begin{gathered}
\frac{d \Psi_{j}}{d X}=i \alpha_{j} \Psi_{q}^{*} \Psi_{p}, \quad \text { where }(j, q)=(m, n) \text { or }(n, m), \\
\frac{d \Psi_{p}}{d X}=i \alpha_{p} \Psi_{m} \Psi_{n},
\end{gathered}
$$

where the constants $\alpha_{m}, \alpha_{n}$, and $\alpha_{p}$ are given by

$$
\begin{gathered}
\alpha_{j}=\frac{-1}{4 k_{j}} \frac{\int_{0}^{H} \phi_{j}(z)\left[B_{q p}(z)+B_{p q}(z)\right] d z}{\int_{0}^{H} \phi_{j}^{2}(z)\left[N^{2}-\omega_{0}^{2}\right] d z}, \text { where }(j, q)=(m, n) \text { or }(n, m), \\
\alpha_{p}=\frac{-1}{4 k_{p}} \frac{\int_{0}^{H} \phi_{p}(z)\left[C_{m n}(z)+C_{n m}(z)\right] d z}{\int_{0}^{H} \phi_{p}^{2}(z)\left[N^{2}-4 \omega_{0}^{2}\right] d z}
\end{gathered}
$$

The expressions for $C_{j q}(z)$ and $B_{j q}(z)$ are $(j=m, n$, or $p$ and $q=m, n$, or $p)$

$$
\begin{aligned}
C_{j q}(z)= & \frac{k_{q}}{\omega_{q}}\left(k_{j}+k_{q}\right)\left(k_{j} N^{2} \phi_{j} \phi_{q}^{\prime}-k_{q} N^{2} \phi_{j}^{\prime} \phi_{q}\right) \\
& -\left(\omega_{j}+\omega_{q}\right)\left(k_{j} \phi_{j}\left(\phi_{q}^{\prime \prime \prime}-k_{q}^{2} \phi_{q}^{\prime}\right)-k_{q} \phi_{j}^{\prime}\left(\phi_{q}^{\prime \prime}-k_{q}^{2} \phi_{q}\right)\right), \\
B_{j q}(z)= & \frac{k_{q}}{\omega_{q}}\left(k_{j}-k_{q}\right)\left(k_{j} N^{2} \phi_{j} \phi_{q}^{\prime}+k_{q} N^{2} \phi_{j}^{\prime} \phi_{q}\right) \\
& -\left(\omega_{j}-\omega_{q}\right)\left(k_{j} \phi_{j}\left(\phi_{q}^{\prime \prime \prime}-k_{q}^{2} \phi_{q}^{\prime}\right)+k_{q} \phi_{j}^{\prime}\left(\phi_{q}^{\prime \prime}-k_{q}^{2} \phi_{q}\right)\right),
\end{aligned}
$$

where $\phi_{m}=\sin (m \pi z / H), \phi_{n}=\sin (n \pi z / H)$, and $\phi_{p}=\sin ((n-m) \pi z / H)$.

Figure 2(a) shows the horizontal spatial evolution of the primary and superharmonic modal amplitudes of the horizontal density gradient perturbation, defined in Eq. (4) based on the numerical solution of Eqs. (20) and (21) for the case-1 resonant triad. The superharmonic wave, extracting energy from the primary waves, grows in amplitude from $x=0$. For small values of $x$, the primary wave amplitudes decrease relatively slowly, resulting in an almost linear growth of the superharmonic wave amplitude. The superharmonic wave amplitude reaches values that are 
significantly larger than the primary wave amplitudes by around $x=0.5 \mathrm{~m}$, beyond which the spatial evolution of all three amplitudes becomes evidently nonlinear. In terms of energy content, by $x=1.66 \mathrm{~m}$, the mode- 4 primary wave has lost all its initial energy, while the mode- 1 superharmonic wave contains $85 \%$ of the total energy. Energy then flows from the superharmonic to the primary waves from $x=1.66 \mathrm{~m}$ to $x=3.32 \mathrm{~m}$, and the relative energy distribution between the three waves at $x=3.32 \mathrm{~m}$ is identical to that at $x=0$. The amplitude evolution between $x=0$ and $x=3.32 \mathrm{~m}$ repeats for larger $x$, assuming that the flow remains in the weakly nonlinear inviscid regime of the isolated resonant triad under consideration. The amplitude evolution for the case- 2 resonant triad [Fig. 2(b)] is qualitatively similar to that for case 1, with the superharmonic mode 2 containing $75 \%$ of the total energy by $x=1.63 \mathrm{~m}$.

In summary, Fig. 2 shows that the superharmonic wave grows linearly with $x$ for small $x$ as the primary wave modes contain finite and slowly varying energies. For typical experimental conditions considered in this paper (primary waves forced at resonance with a horizontal displacement amplitude of $5 \mathrm{~mm}$ each), the superharmonic wave is predicted to contain the bulk of the total energy by around $x=1.6 \mathrm{~m}$. In our experiments, we aim to validate the theoretical predictions presented in Fig. 2. As discussed in Sec. IV, the spatial growth at small $x$ is rigorously validated by experiments. The longer term spatial evolution, however, is not so easily validated due to viscous effects and the end walls of the tank generating a reflected wave field in our measurement region. The viscous effects, including a methodology on how to incorporate them in theory, are discussed in Sec. V B.

\section{RESULTS}

In this section, we present our experimental results obtained for the case- 1 and case- 2 resonant triads. Sections IV A-IV D concern the case-1 resonant triad, sequentially showing the generation of a superharmonic wave field, validation of the theoretically predicted spatial structure and the amplitude evolution equations at resonance, investigation of the superharmonic wave field at off-resonant frequencies, and finally a presentation of results on the effect of the primary wave forcing amplitudes. Section IV E concerns the case-2 resonant triad, demonstrating the occurrence of resonance, validating the theoretical amplitude evolution equations, and studying the off-resonant behavior.

\section{A. Superharmonic wave generation (case-1 resonant triad)}

Figure 3 shows the time frequency plots based on the measured horizontal density gradient field from four different experiments with the forcing corresponding to $(m, n)=(3,4)$. Spectral peaks whose widths $\Delta \omega$ are around $0.3 \omega$ are observed, thus requiring a frequency window of a larger width (specifically chosen as $0.4 \omega$ in the current study) for calculating filtered wave fields as mentioned in Sec. II. Figure 3(a), corresponding to the experiment with $\left(A_{3}, A_{4}\right)=(5,5) \mathrm{mm}$ and the resonant forcing frequency of $\omega_{0} / N=0.4841$, shows the emergence of a strong signal at the superharmonic frequency $2 \omega_{0}$. By around $10 T_{0}$, the wave field at $2 \omega_{0}$ seems to be as strong as the wave field at the forcing frequency $\omega_{0}$. In contrast, at the off-resonant forcing frequency of $\omega_{0} / N=0.3$ [Fig. 3(b)], the superharmonic generation is significantly weaker, with a strength that is at least a couple of orders of magnitude smaller than the primary wave field. It is noteworthy that the forcing amplitudes are $\left(A_{3}, A_{4}\right)=(5,5) \mathrm{mm}$ in Fig. 3(b), too, and hence the difference between Figs. 3(a) and 3(b) is attributed entirely to the difference in the forcing frequencies. The weak superharmonic wave field in Fig. 3(b) could still be a result of off-resonant wave-wave interaction between the primary modes, as suggested by the constant-amplitude theory in Sec. III A.

To highlight the role of simultaneous presence of modes $m$ and $n$ in the primary wave field for resonant superharmonic generation, the bottom row of Fig. 3 shows results from experiments where only mode 3 [Fig. 3(c)] or mode 4 [Fig. 3(d)] was forced, both at the resonant forcing frequency of $\omega_{0} / N=0.4841$. Both experiments show negligible generation of a superharmonic wave field, thus 


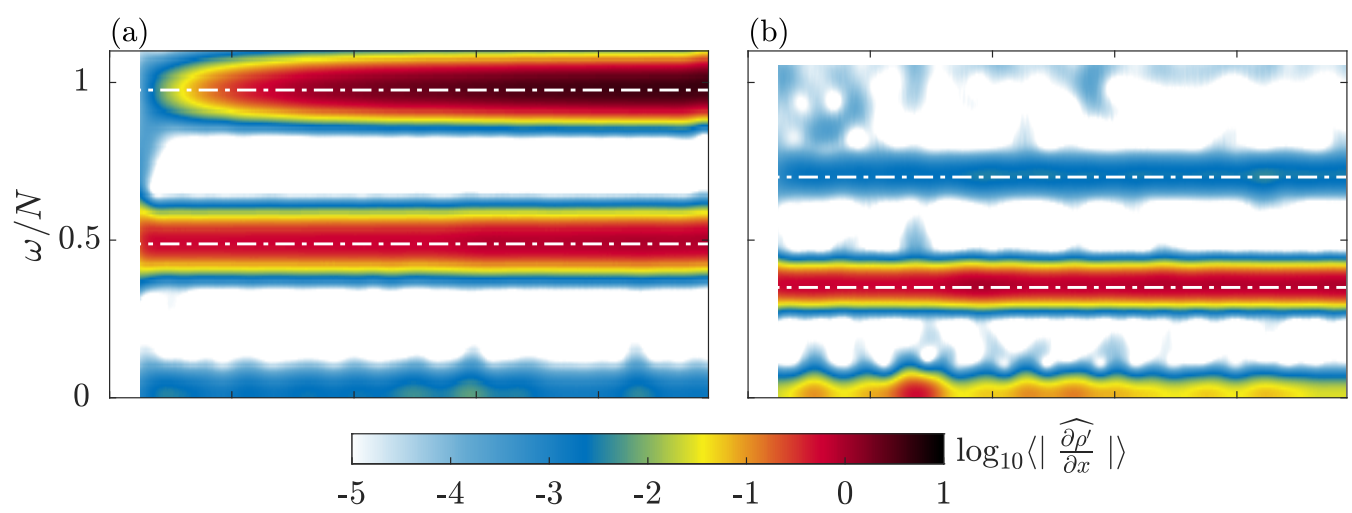

(c)
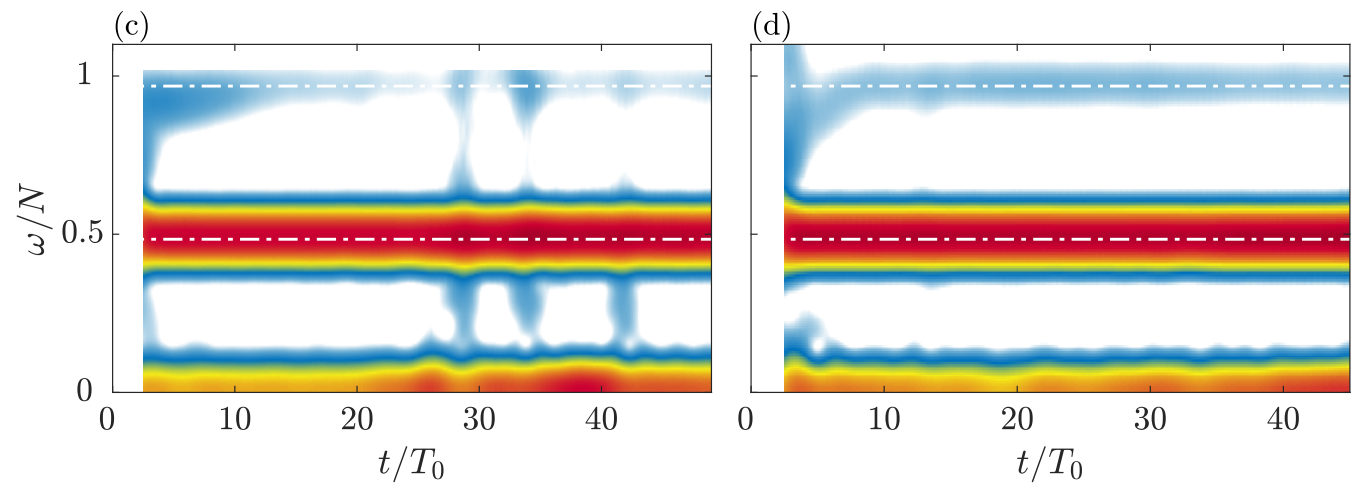

FIG. 3. Time frequency spectrum $\left|\widehat{\partial \rho^{\prime} / \partial x}(\omega, t)\right|$, spatially averaged over $6 \leqslant x \leqslant 25 \mathrm{~cm}, 0 \leqslant z \leqslant$ $32.5 \mathrm{~cm}$, in four different experiments for the case-1 resonant triad, i.e., $(m, n, p)=(3,4,1)$. The four panels correspond to (a) the resonant forcing frequency $\omega_{0} / N=0.4841$ with $\left(A_{3}, A_{4}\right)=(5,5) \mathrm{mm}$, (b) the off-resonant forcing frequency $\omega_{0} / N=0.3$ with $\left(A_{3}, A_{4}\right)=(5,5) \mathrm{mm}$, (c) the resonant forcing frequency $\omega_{0} / N=0.4841$ with $\left(A_{3}, A_{4}\right)=(5,0) \mathrm{mm}$, and (d) the resonant forcing frequency $\omega_{0} / N=0.4841$ with $\left(A_{3}, A_{4}\right)=(0,5) \mathrm{mm}$. The white dashed-dotted lines in all the plots correspond to $\omega / N=\omega_{0} / N$ and $2 \omega_{0} / N$. Every plot has been normalized such that the maximum value at the forcing frequency $\omega=\omega_{0}$ is unity.

confirming that the interaction between modes 3 and 4 is essential for the superharmonic generation observed in Fig. 3(a). Furthermore, Figs. 3(c) and 3(d) show that excitation of a superharmonic wave field, if any, by the wave generator itself is negligible. Additionally, noticeable mean flow seems to occur in all the cases where resonant superharmonic generation is absent, i.e., Figs. 3(b)-3(d), an aspect we will pursue in future studies. In summary, Fig. 3 presents experimental confirmation of strong superharmonic generation due to resonant interaction between modes 3 and 4 at the primary wave frequency of $\omega_{0} / N=0.4841$. We proceed to investigate the spatial structure of the superharmonic wave field observed in Fig. 3(a), and verify if it indeed contains predominantly mode $p$ with $p=|m-n|=1$.

\section{B. Forcing at resonant frequency (case-1 resonant triad)}

Figure 4(a) shows the spatial structure of the steady-state wave field filtered at the forcing frequency $\omega_{0}$ in the experiment corresponding to Fig. 3(a), i.e., $(m, n)=(3,4),\left(A_{3}, A_{4}\right)=(5,5)$ $\mathrm{mm}$, and $\omega_{0} / N=0.4841$. For reference, we recall from Sec. II that the horizontal wavelengths of the primary modes are $\lambda_{3}=0.392 \mathrm{~m}, \lambda_{4}=0.294 \mathrm{~m}$, and that of the superharmonic mode is $\lambda_{1}=0.168$ $\mathrm{m}$. The observed wave field can be understood as a superposition of spatially evolving modes 3 and 4 that are forced at $x=0$. Indeed, modal decomposition at different $x$ locations reveals that the 

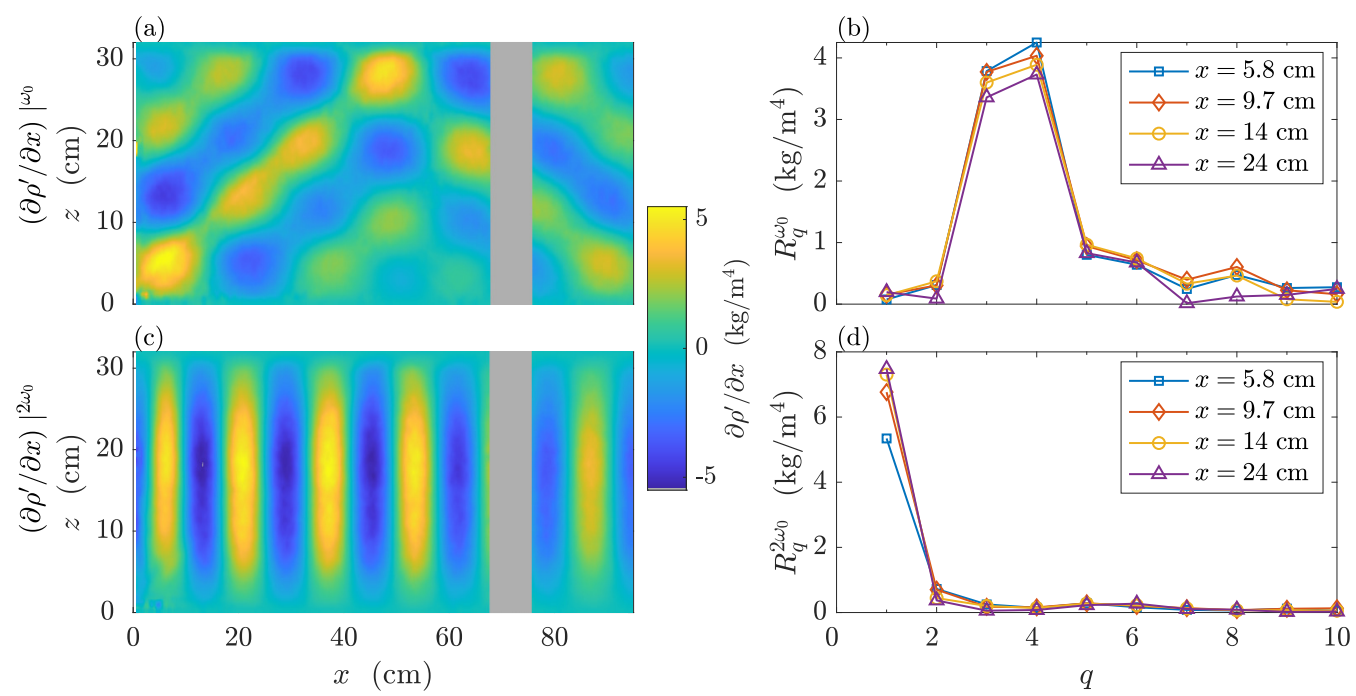

FIG. 4. Experimental measurements for the case-1 resonant triad, i.e., $(m, n, p)=(3,4,1)$, at the resonant forcing frequency of $\omega_{0} / N=0.4841$, and forcing amplitudes of $\left(A_{3}, A_{4}\right)=(5,5) \mathrm{mm}$. (a) Horizontal density gradient field filtered at the forcing frequency $\omega_{0}$. (b) Modal decomposition at four different $x$ locations (see legend) of the wave field shown in (a). (c) Horizontal density gradient field filtered at twice the forcing frequency, i.e., $2 \omega_{0}$. (d) Modal decomposition at four different $x$ locations (see legend) of the wave field shown in (c). All the plots correspond to $t=20 T_{0}$, thus representing the steady-state wave field. The gray regions in (a) and (c) correspond to the joint region indicated by the first black strip in Fig. 1, where measurements were not possible.

steady-state wave field predominantly contains modes 3 and 4 [Fig. 4(b)]. Furthermore, we also observe that the primary wave amplitudes decay with $x$, with modes 3 and 4 reducing in amplitude by $13.8 \%$ and $11.5 \%$, respectively, over $0.182 \mathrm{~m}$. The occurrence of weak but nonzero amplitudes at modes 5 and 6 is attributed to both the discrete nature of forcing by the wave generator, and finite resolution (in space and time) of the experimental measurements.

Figure 4(c) shows the spatial structure of the steady-state wave field filtered at the superharmonic frequency $2 \omega_{0}$ in the same experiment as in Fig. 4(a). A mode- 1 structure is clearly evident, and is consistent with the theoretical prediction that the generated superharmonic wave will have a mode- $p$ structure with $p=|m-n|=1$. Furthermore, the observed horizontal wavelength of $15.5 \mathrm{~cm}$ is close to the theoretical estimate of $16.8 \mathrm{~cm}$ for the mode- 1 internal wave horizontal wavelength at $2 \omega_{0} / N$ (see end of Sec. II). The results from modal decomposition [Fig. 4(d)] confirm that the superharmonic wave field is dominated by mode 1 , with its amplitude increasing with $x$.

We proceed to compare the observed spatial growth of the steady-state superharmonic modal amplitude in the resonant forcing frequency experiment with the theoretical predictions based on the amplitude evolution Eqs. (20) and (21). Figure 5(a) shows the spatial evolution of the steady-state primary wave modal amplitudes in the case- 1 resonant triad experiment with $\left(A_{3}, A_{4}\right)=(5,5)$ $\mathrm{mm}$ and the resonant forcing frequency of $\omega_{0} / N=0.4841$. In other words, we perform modal decomposition at all $x$ in the wave field shown in Fig. 4(a) to plot $R_{3}^{\omega_{0}}$ and $R_{4}^{\omega_{0}}$ as a function of $x$. The observed mode- 3 and mode- 4 primary wave amplitudes show a decay of $25 \%$ and $24.7 \%$, respectively, over $50 \mathrm{~cm}$; corresponding values based on the inviscid amplitude evolution equations (solutions plotted using solid lines) are $6.8 \%$ and $12.9 \%$. We attribute the relatively stronger observed decay to viscous effects, an aspect that is discussed further in Sec. V B. The superharmonic mode- 1 amplitude, normalized by $R_{3}^{\omega_{0}} R_{4}^{\omega_{0}}$ measured at $x=5.5 \mathrm{~cm}$, shows an almost linear growth until around $x \simeq 40 \mathrm{~cm}$, beyond which it shows an evidently nonlinear evolution 

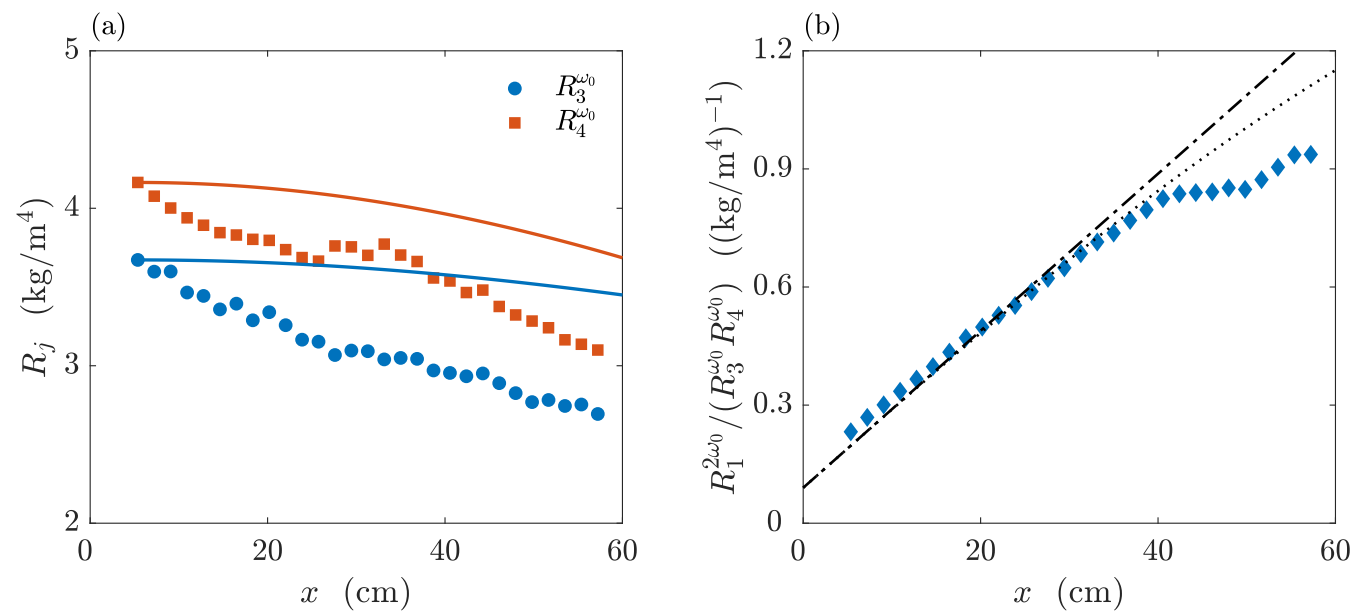

FIG. 5. Spatial evolution of the steady-state modal amplitudes at resonance for the case-1 resonant triad experiment shown in Fig. 4. (a) Observed spatial evolution of the primary wave modal amplitudes, $R_{3}^{\omega_{0}}$ and $R_{4}^{\omega_{0}}$. Predictions from the inviscid amplitude evolution Eqs. (20) and (21), with initial conditions taken from measured primary wave amplitudes at $x=5.5 \mathrm{~cm}$, are shown using solid lines. (b) Spatial evolution of the superharmonic mode- $p=|m-n|=1$ amplitude $R_{1}^{2 \omega_{0}}$, normalized by $R_{3}^{\omega_{0}} R_{4}^{\omega_{0}}$ measured at $x=5.5 \mathrm{~cm}$. The black dashed-dotted line indicates theoretical linear growth for small $x$, based on Eq. (27). The dotted line indicates the numerical solution of Eqs. (20) and (21). All the experimental data shown in this figure are based on the measurements between $20 T_{0}$ and $25 T_{0}$.

[Fig. 5(b)]. To understand the linear growth, one can integrate Eq. (21) to write

$$
\Psi_{p}(X)=\Psi_{p}(X=0)+i \alpha_{p} \Psi_{m}(X=0) \Psi_{n}(X=0) X,
$$

where we assume that $\Psi_{m}(X)$ and $\Psi_{n}(X)$ remain invariant with $X$. Indeed, in the inviscid limit, this assumption is valid for small $X$, as seen in the numerical solution of the amplitude evolution equations in Fig. 2. Writing in terms of the actual wave field (recall that $\epsilon \psi_{1}$ is what represents the primary wave field) and the physical coordinate $x$ (recall that $X=\epsilon x$ ), Eq. (26) becomes

$$
\tilde{\Psi}_{p}(x)=\tilde{\Psi}_{p}(x=0)+i \alpha_{p} \tilde{\Psi}_{m}(x=0) \tilde{\Psi}_{n}(x=0) x,
$$

where $\tilde{\Psi}_{j}=\epsilon \Psi_{j}$ for $j=m, n$, or $p$. Therefore, with $\tilde{\Psi}_{p}(x=0)=0, \tilde{\Psi}_{p}(x) /\left(\tilde{\Psi}_{m}(x=0) \tilde{\Psi}_{n}(x=0)\right)$ goes as $i \alpha_{p} x$, with the normalization by $\tilde{\Psi}_{m}(x=0) \tilde{\Psi}_{n}(x=0)$ allowing us to isolate the effects of forcing frequency from the forcing amplitudes. The resulting theoretical slope for the linear growth of $R_{1}^{2 \omega_{0}} /\left(R_{3}^{\omega_{0}} R_{4}^{\omega_{0}}\right)$ accurately captures the experimental observation for $x$ values at least until around $40 \mathrm{~cm}$, as shown by the dashed dotted line in Fig. 5(b). Here, the normalizing factor $R_{3}^{\omega_{0}} R_{4}^{\omega_{0}}$ is taken as what is measured at $x=5.5 \mathrm{~cm}$, which is the closest location to the wave generator where the measured primary wave field is without any noticeable noise. Due to unknown complex processes occurring close to the wave generator $(x<5 \mathrm{~cm})$, the exact physical location of the origin of the $x$ axis is unknown, thus yielding an apparent nonzero intercept in Fig. 5(b). An example of such a process is the possibility of the forced primary waves generating a superharmonic wave field in the near vicinity of the wave generator akin to the constant-amplitude theory in Sec. III A. In summary, Fig. 5 quantitatively validates the theoretical prediction of linear growth of the steady-state superharmonic mode- 1 amplitude for sufficiently small $x$. However, the relatively stronger decay in the observed primary wave amplitudes [Fig. 5(a)], and the nonlinear features in the superharmonic wave field for $x>40 \mathrm{~cm}$ [Fig. 5(b)] are in contrast to the fully numerical solution of the inviscid amplitude evolution equations [Fig. 2(a)]. While the viscous wave field evolution over 


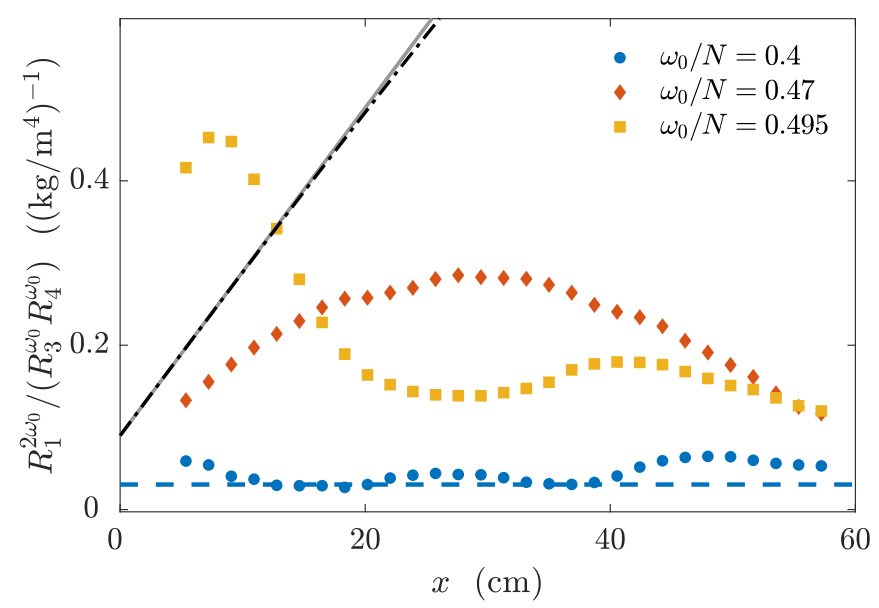

FIG. 6. Spatial evolution of the normalized steady-state superharmonic mode-1 amplitude at off-resonant forcing frequencies for the case-1 resonant triad, i.e., $(m, n, p)=(3,4,1)$. All three experiments in this plot were performed with $\left(A_{3}, A_{4}\right)=(5,5) \mathrm{mm}$ at forcing frequencies indicated in the legend. The blue dashed line indicates the superharmonic mode- 1 amplitude for $\omega_{0} / N=0.4$ based on the constant-amplitude solution in Sec. III A. The gray solid line indicates theoretical linear growth for small $x$ at resonance based on Eq. (27). The black dashed-dotted line indicates the numerical solution of Eqs. (20) and (21). The normalizing factor is $R_{3}^{\omega_{0}} R_{4}^{\omega_{0}}$ as measured at $x=5.5 \mathrm{~cm}$ in the corresponding experiment.

larger $x$ is discussed in Sec. VB, we recall that reflected waves from the right end of the tank make it difficult to observe the steady-state wave field at large $x$.

\section{Forcing at off-resonant frequencies (case-1 resonant triad)}

Modal decomposition and spatial evolution analyses such as in Figs. 4 and 5(b) are performed for all off-resonant forcing frequency experiments to investigate the frequency extent (around the resonant frequency) over which superharmonic wave generation occurs. It is, however, worth highlighting that the superharmonic wave need not be an internal wave if the forcing is away from the resonant frequency. In other words, at off-resonance, what we obtain as mode 1 at $2 \omega_{0}$ may not satisfy the dispersion relation for internal wave modes.

As a result of the modal analysis, Fig. 6 shows the spatial evolution of steady-state normalized superharmonic mode- $p=1$ amplitude from experiments at three different off-resonant frequencies (indicated in the legend). Far from resonance, i.e., $\omega_{0} / N=0.4$, superharmonic wave generation is observed to be small. The normalized superharmonic mode- 1 amplitude for $\omega_{0} / N=0.4$ based on the constant-amplitude solution in Sec. III A (blue dashed line) provides a mechanism for superharmonic wave generation far from resonance. At a forcing frequency closer to resonance, i.e., $\omega_{0} / N=0.47$, a somewhat linear growth is observed up to around $20 \mathrm{~cm}$, albeit with a slope smaller than what is observed at resonance (gray solid line/black dashed-dotted line). Furthermore, the superharmonic wave amplitude decays beyond $x=30 \mathrm{~cm}$ for $\omega_{0} / N=0.47$, which is in contrast to what we observe at resonance in Fig. 5(b). For $\omega_{0} / N=0.495$, which is larger than the resonant frequency, we observe relatively weak superharmonic waves for $x>20 \mathrm{~cm}$ when compared to $\omega_{0} / N=0.47$. For $x<20 \mathrm{~cm}$, however, relatively strong superharmonic waves are observed for $\omega_{0} / N=0.495$. We attribute this occurrence to the possibility of other resonant interactions generating superharmonic mode 1 . The presence of weak modes 5 and 6 in the primary wave field [see Fig. 4(b)] may result in the resonant generation of superharmonic mode 1 due to the interaction between mode 4 and mode 5 , or mode 5 and mode 6 ; the resonant forcing frequencies for $(m, n, p)=$ $(4,5,1)$ and $(5,6,1)$ are $\omega_{0} / N=0.4905$ and 0.4937 , respectively, therefore relatively close to 
(a)

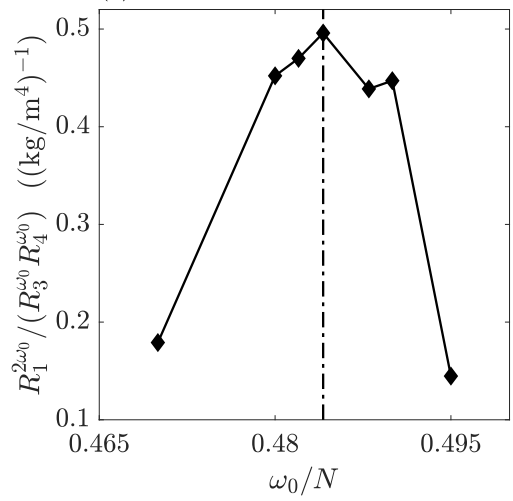

(b)

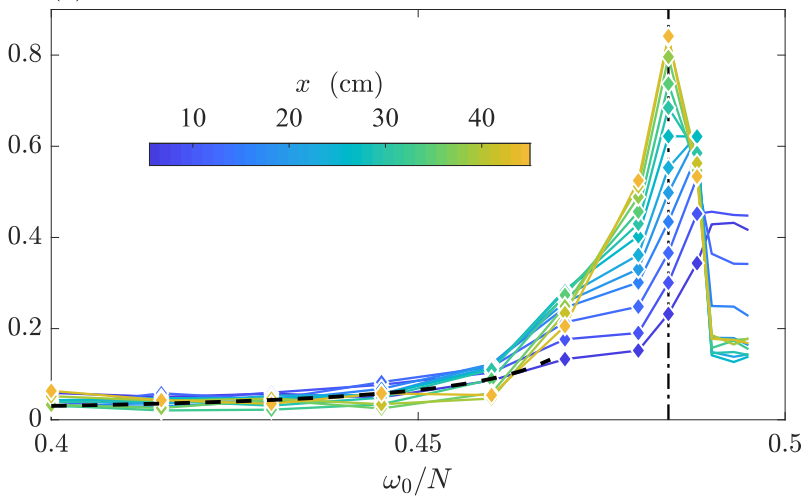

FIG. 7. (a) Normalized steady-state superharmonic mode-1 amplitude at $x=25 \mathrm{~cm}$ as a function of the forcing frequency $\omega_{0}$ for the case-1 resonant triad, i.e., $(m, n, p)=(3,4,1)$. (b) Normalized steady-state superharmonic mode-1 amplitude at various $x$ (see legend) as a function of the forcing frequency $\omega_{0}$ for the case-1 resonant triad, i.e., $(m, n, p)=(3,4,1)$, over a wider range of $\omega_{0}$ than in (a). The black dashed line represents the normalized superharmonic amplitude based on the constant-amplitude solution in Sec. III A. In both (a) and (b), the normalizing factor is $R_{3}^{\omega_{0}} R_{4}^{\omega_{0}}$ as measured at $x=5.5 \mathrm{~cm}$ in the corresponding experiment. The vertical dashed dotted line in both plots is drawn at the theoretical resonant forcing frequency of $\omega_{0} / N=0.4841$.

0.495. The theoretical slope of linear growth of $R_{p}^{2 \omega_{0}} /\left(R_{m}^{\omega_{0}}(x=0) R_{n}^{\omega_{0}}(x=0)\right)$ at small $x$ [Eq. (27)] for $(m, n, p)=(3,4,1),(4,5,1)$, and $(5,6,1)$ are $1.28,2.15$, and $3.24 \mathrm{~m}^{3} / \mathrm{kg}$, respectively. This suggests that other resonant interactions at larger $(m, n)$, and hence larger $\omega_{0} / N$ for resonance based on Eq. (2), could result in relatively faster growth of the superharmonic mode- 1 amplitude at small $x$ even if the primary wave amplitudes are relatively weak. Furthermore, for $\omega_{0} / N=0.495$, the superharmonic frequency is very close to evanescent behavior, and the corresponding enhanced viscous decay (see Sec. V B) could explain why the superharmonic mode 1 rapidly decays.

We proceed to explore several other off-resonant frequencies, and compute the steady-state normalized superharmonic mode-1 amplitude at a fixed $x$ location for several $\omega_{0} / N$ around the resonant frequency. It is noteworthy that constant horizontal displacement amplitudes $\left(A_{3}=\right.$ $A_{4}=5 \mathrm{~mm}$ is forced at all frequencies) do not correspondingly lead to the same amplitudes at different frequencies for the horizontal density gradient, thus motivating the normalization of the superharmonic wave amplitude. Figure 7(a) shows $R_{1}^{2 \omega_{0}} /\left(R_{3}^{\omega_{0}} R_{4}^{\omega_{0}}\right)$ at $x=25 \mathrm{~cm}$ as a function of $\omega_{0} / N$. A clear peak is observed very close to $\omega_{0} / N=0.4841$, thus confirming the occurrence of resonance at the theoretically predicted forcing frequency. Normalization of the superharmonic amplitude eliminates any role for the forcing primary amplitudes in the occurrence of the resonant peak. In Fig. 7(b), we present a similar plot as in Fig. 7(a), but for a wider range of $\omega_{0} / N$ and several $x$ locations (see legend). A resonant peak at the theoretical prediction of $\omega_{0} / N=0.4841$ is present for all $x>24 \mathrm{~cm}$, with the peak being slightly shifted to the right for $x<24 \mathrm{~cm}$. As discussed earlier for Fig. 6, relatively strong superharmonic mode-1 generation at small $x$ for $\omega_{0} / N>0.4841$ could be due to other resonant interactions involving mode 5 or 6 that are present in the forced primary wave field. At forcing frequencies much smaller than the resonant frequency, small but finite superharmonic mode- 1 amplitude is observed, which is explained by the constant-amplitude superharmonic solution (black dashed line) obtained based on off-resonant interaction between primary mode 3 and mode 4 . In summary, Fig. 7 presents experimental demonstration of resonant generation of superharmonic mode 1 at $\omega_{0} / N=0.4841$ for $(m, n, p)=(3,4,1)$, with the resonance peak being around $\Delta\left(\omega_{0} / N\right)=0.03$ in width (approximately estimated by visual inspection). 
(a)

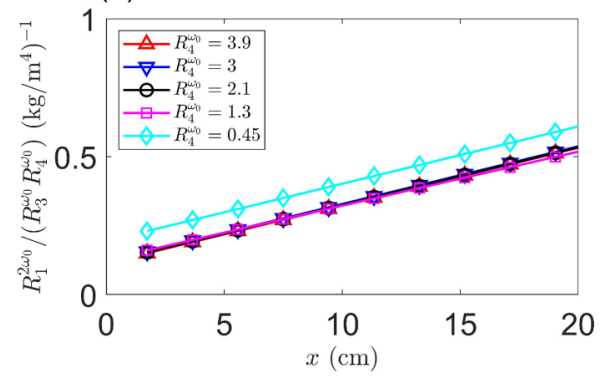

(b)

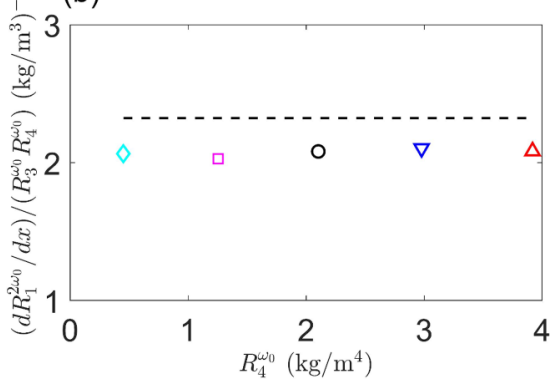

FIG. 8. Effects of forcing amplitude for the case-1 resonant triad at resonance, i.e., $(m, n, p)=(3,4,1)$ and $\omega_{0} / N=0.4841$. (a) Spatial evolution of the normalized steady-state superharmonic mode- 1 amplitude for different mode-4 forcing amplitudes $\left(A_{4}=5,4,3,2,1 \mathrm{~mm}\right.$, with the corresponding measured $R_{4}^{\omega_{0}}$ at $x=$ $6 \mathrm{~cm}$ indicated in the legend), while the mode- 3 forcing amplitude was held fixed at $A_{3}=5 \mathrm{~mm}$. The solid lines indicate the best fit straight line for the corresponding experiment. (b) Variation of the slope of the best fit straight lines from (a), i.e., the spatial growth rate of the normalized steady-state superharmonic mode-1 amplitude, with the mode- 4 forcing amplitude. The dashed line indicates the theoretical prediction based on the amplitude evolution equations. In both (a) and (b), the normalizing factor is $R_{3}^{\omega_{0}} R_{4}^{\omega_{0}}$ as measured at $x=6$ $\mathrm{cm}$ in the corresponding experiment.

\section{Effect of forcing amplitude at resonance (case-1 resonant triad)}

In this subsection, we present results from experiments at resonance $\left(\omega_{0} / N=0.4841\right)$ for the case-1 resonant triad, i.e., $(m, n, p)=(3,4,1)$, with varying $A_{4}$ while $A_{3}$ was held fixed at $5 \mathrm{~mm}$. As shown in Fig. 8(a), linear growth of the normalized steady-state superharmonic mode- 1 amplitude is observed for small $x$ in every experiment, with the legend indicating the measured $R_{4}^{\omega_{0}}$ at $x=5.5 \mathrm{~cm}$. As expected based on Eq. (27), the slope of the linear growth hardly changes as $R_{4}^{\omega_{0}}$ is varied. The experiment with the weakest forcing amplitude $\left(R_{4}^{\omega_{0}}=0.45 \mathrm{~kg} / \mathrm{m}^{4}\right)$ falls above the rest of the experiments, probably owing to errors in the measurement of small $R_{4}^{\omega_{0}}$ at $x=6 \mathrm{~cm}$ that is used in the normalization of the superharmonic wave amplitude. A plot of the measured slope [from Fig. 8(a)] as a function of $R_{4}^{\omega_{0}}$ is in fair agreement with the theoretical prediction shown using the dashed horizontal line in Fig. 8(b). The observed spatial growth of the normalized superharmonic mode- 1 amplitude is weaker (by around 10\%) than the inviscid theoretical estimate across all values of primary mode- 4 amplitude. This could be due to viscous effects, including those at the side walls of the tank, which we discuss further in Sec. V B. In summary, Fig. 8 provides further quantitative validation of the amplitude evolution in Eq. (21) at small $x$.

\section{E. Case-2 resonant triad}

To demonstrate the robustness of our experimental results, and subsequent quantitative validation of the amplitude evolution equations, we performed experiments for another resonant triad. The case- 2 resonant triad is specified by $(m, n, p)=(3,5,2)$, whose resonant forcing frequency according to Eq. (2) is $\omega_{0} / N=0.4472$. Based on plots similar to Figs. 7(a) and 7(b) (not shown) with $\left(A_{3}, A_{5}\right)=(5,5) \mathrm{mm}$, we observe a resonance peak at around $\omega_{0} / N=0.46$. The small shift in the resonant frequency from the theoretical prediction could be due to the weak presence of other resonantly interacting modes in the forced primary wave field. For example, the resonant forcing frequency for $(m, n, p)=(4,6,2)$ is $\omega_{0} / N=0.4677$.

Figure 9 shows the spatial structure of the steady-state wave field filtered at $\omega_{0}$ (top row) and $2 \omega_{0}$ (bottom row) in the case- 2 resonant triad experiment at the measured resonant forcing frequency of $\omega_{0} / N=0.46$. For reference, we recall from Sec. II that the horizontal wavelengths of the primary modes are $\lambda_{3}=0.433 \mathrm{~m}, \lambda_{5}=0.26 \mathrm{~m}$, and that of the superharmonic mode is $\lambda_{2}=0.163 \mathrm{~m}$. Indeed, a modal decomposition of the wave field in Fig. 9(a) reveals amplitudes 

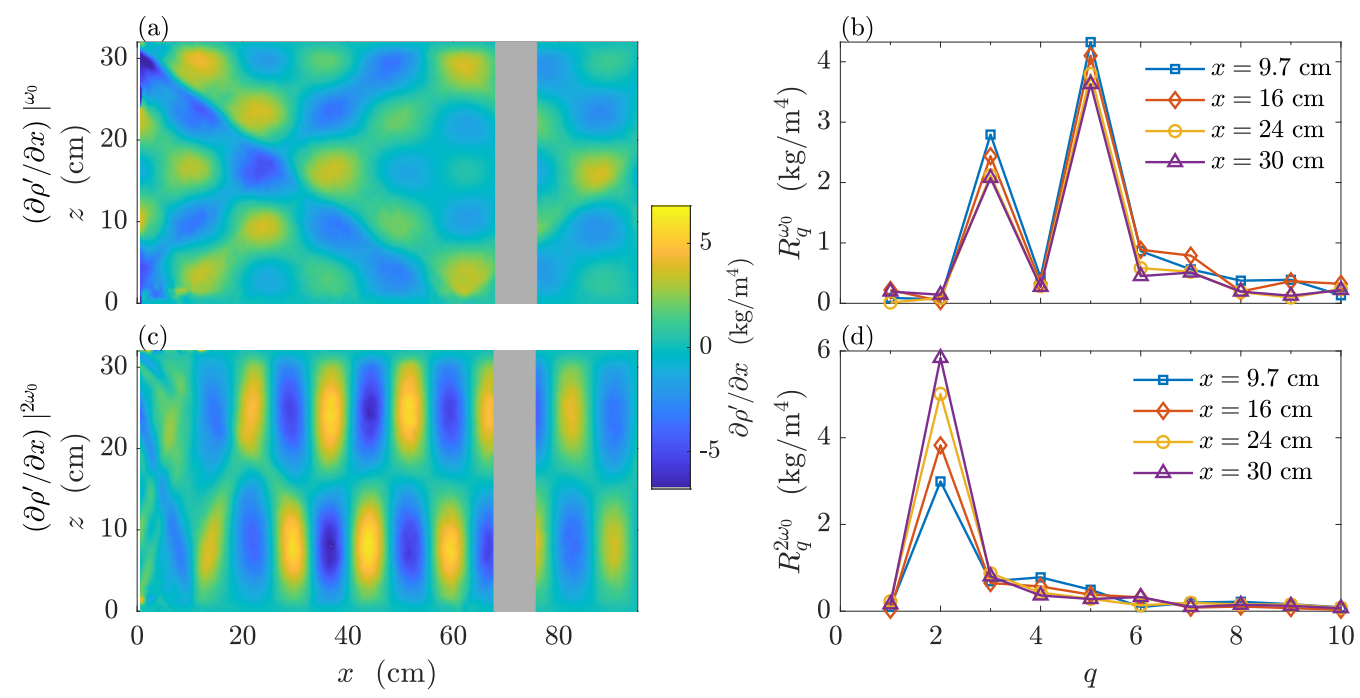

FIG. 9. Experimental measurements for the case-2 resonant triad, i.e., $(m, n, p)=(3,5,2)$, at the measured resonant forcing frequency of $\omega_{0} / N=0.46$, and forcing amplitudes of $\left(A_{3}, A_{5}\right)=(5,5) \mathrm{mm}$. (a) Horizontal density gradient field filtered at the forcing frequency $\omega_{0}$. (b) Modal decomposition at four different $x$ locations (see legend) of the wave field shown in (a). (c) Horizontal density gradient field filtered at twice the forcing frequency, i.e., $2 \omega_{0}$. (d) Modal decomposition at four different $x$ locations (see legend) of the wave field shown in (c). All the plots correspond to $t=20 T_{0}$, thus representing the steady-state wave field.

predominantly at mode 3 and mode 5, though relatively weak amplitudes are present at other modes as well [Fig. 9(b)]. Weak spatial decay is observed in the amplitudes of both mode 3 and mode 5 , which we attribute to viscous effects. The steady-state superharmonic wave field shows a clearly predominant mode-2 spatial structure [Fig. 9(c)], which is consistent with the resonant generation of mode $p=|m-n|=2$. The modal decomposition in Fig. 9(d) shows a spatial growth of the mode-2 amplitude in the steady-state superharmonic wave field, which we compare with the prediction from the amplitude evolution equations in Fig. 10.

Figure 10 shows that the spatial growth of the normalized, steady-state mode- 2 amplitude at the measured resonant forcing frequency of $\omega_{0} / N=0.46$ is predicted accurately until around $x=35 \mathrm{~cm}$ by the theoretical linear growth at small $x$ in Eq. (27). For smaller forcing frequencies, spatial linear growth of the normalized steady-state mode 2 is observed at small $x$, with progressively smaller slopes as we move away from resonance. For $\omega_{0} / N>0.46$, relatively large mode- 2 amplitude is observed for very small $x$, before rapid decay of the same for larger $x$. Similar to the proposed mechanism for other resonant interactions generating mode 1 for large $\omega_{0} / N$ in the case-1 resonant triad (Fig. 6), it is possible that resonant interaction between relatively weak mode 4 and mode 6 , or mode 5 and relatively weak mode 7 in the forced primary wave field may generate superharmonic mode 2 . For resonant triads specified by $(m, n, p)=(4,6,2)$ and $(m, n, p)=(5,7,2)$, the resonant forcing frequencies are $\omega_{0} / N=0.4677$ and 0.4781 , respectively. The theoretical slope of linear growth of $R_{2}^{2 \omega_{0}} /\left(R_{m}^{\omega_{0}}(x=0) R_{n}^{\omega_{0}}(x=0)\right)$ at small $x$ [Eq. (27)] for $(m, n, p)=(3,5,2),(4,6,2)$, and $(5,7,2)$ are $0.75,1.25$, and $1.84 \mathrm{~m}^{3} / \mathrm{kg}$, respectively. This suggests that other resonant interactions at larger $(m, n)$, and hence larger $\omega_{0} / N$ for resonance based on Eq. (2), could result in relatively rapid growth of the superharmonic mode-2 amplitude at small $x$ even if the primary wave amplitudes are relatively weak. Furthermore, the enhanced viscous effects at these large $2 \omega_{0} / N$ may also result in rapid decay of the superharmonic mode-2 amplitude (Sec. V B). 


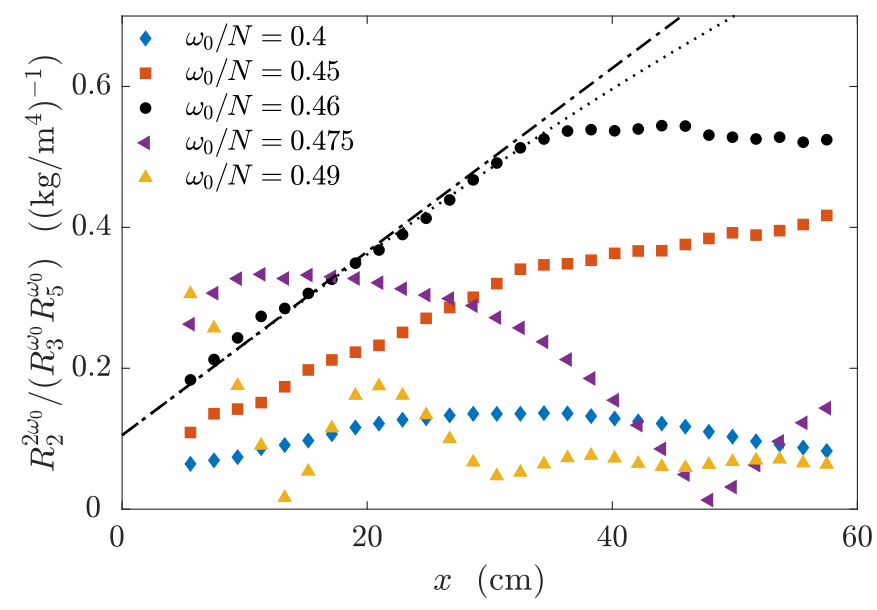

FIG. 10. Spatial evolution of the normalized steady-state superharmonic mode- 2 amplitude at resonant $\left(\omega_{0} / N=0.46\right)$ and off-resonant forcing frequencies for the case-2 resonant triad, i.e., $(m, n, p)=(3,5,2)$. All five experiments in this plot were performed with $\left(A_{3}, A_{5}\right)=(5,5) \mathrm{mm}$ at forcing frequencies indicated in the legend. The normalizing factor is $R_{3}^{\omega_{0}} R_{5}^{\omega_{0}}$ as measured at $x=5.5 \mathrm{~cm}$ in the corresponding experiment. The black dashed-dotted line indicates theoretical linear growth for small $x$ at resonance based on Eq. (27). The dotted line indicates the numerical solution of Eqs. (20) and (21).

In this subsection, we have presented an experimental demonstration of resonant generation of superharmonic mode 2 for the case- 2 resonant triad, i.e., $(m, n, p)=(3,5,2)$. The observed spatial growth at small $x$ of the superharmonic mode 2 in the steady-state wave field is well captured by the theoretical amplitude evolution equations. Finally, experiments at off-resonant forcing frequencies revealed that the superharmonic mode- 2 generation becomes weaker as we move away from resonance, whose frequency width is observed to be a similar value as that for the case- 1 resonant triad, i.e., $\Delta \omega_{0} \approx 0.03 N$.

\section{DISCUSSION}

In Sec. IV, we reported experimental observations of superharmonic generation due to triadic resonance, and validated the amplitude evolution equations in describing the spatial growth of the steady-state superharmonic wave. In this section, we investigate two important questions that were not addressed in the previous section. In Sec. V A, the initial temporal growth of the superharmonic wave at a fixed horizontal location (before it reaches steady state) is investigated. The role of viscous effects in our experimental observations is then discussed in Sec. V B.

\section{A. Slow temporal evolution}

The spatial growth of the steady-state superharmonic wave at small distances from the wave generator is captured accurately by the amplitude evolution equations, (20) and (21), wherein it was assumed that the steady-state modal amplitudes evolve over relatively large spatial scales. During the transient period in the experiment, however, the wave amplitudes at a fixed spatial location are temporally evolving, thus rendering the amplitude evolution equations, (20) and (21), not well suited. We proceed to explore how well amplitude evolution equations based on a slow temporal evolution could describe the observed wave fields before steady state is reached.

In contrast to the large scale spatial evolution assumed in Sec. III B, previous studies [22,35] on triadic resonance have used a framework which assumes a slow temporal evolution of the amplitudes of each of the waves forming the resonant triad. Defining the slow time $\tau=\epsilon t$, the wave field at 

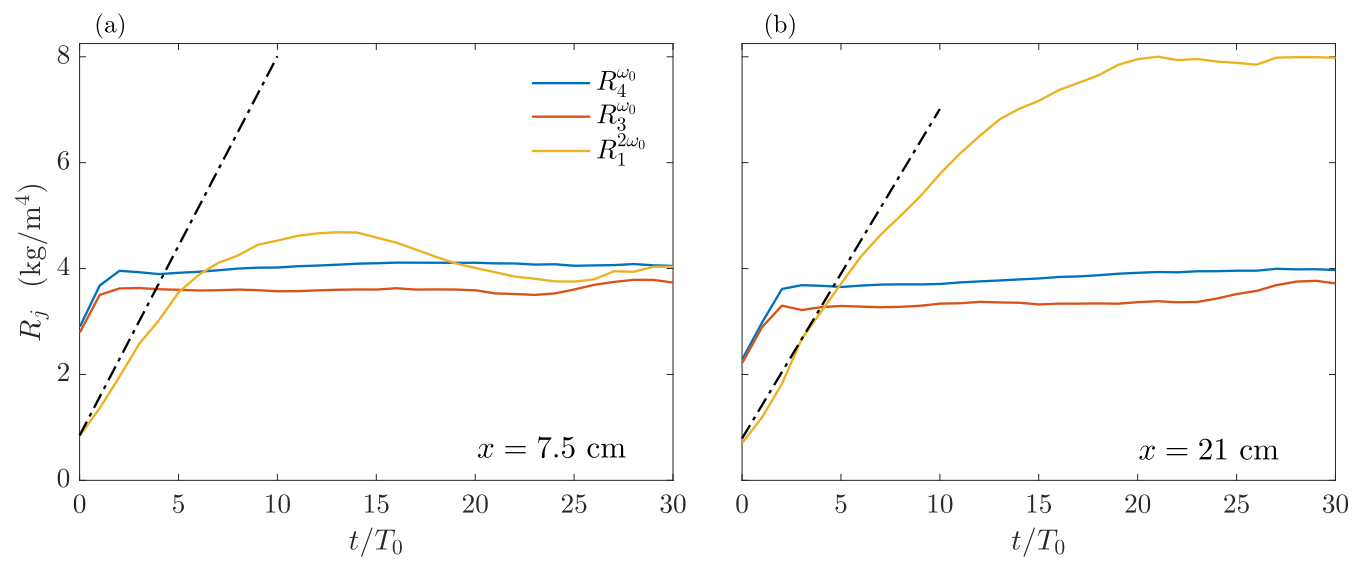

FIG. 11. Temporal evolution of the modal amplitudes at a fixed $x$ location for the case-1 resonant triad at resonance, i.e., $(m, n, p)=(3,4,1)$ at $\omega_{0} / N=0.4841$, with $\left(A_{m}, A_{n}\right)=(5,5) \mathrm{mm}$. (a) $x=7.5 \mathrm{~cm}$, and (b) $x=21 \mathrm{~cm}$. The dashed-dotted lines in (a) and (b) indicate predictions of initial growth from the slow temporal evolution theory presented in Sec. V A, using the measured steady-state primary wave amplitudes at the corresponding $x$ as initial conditions for the growth of the superharmonic wave.

$O(\epsilon)$ is written as

$$
\begin{aligned}
\Psi_{m}(x, z, t)= & \frac{\Psi_{m}(\tau)}{2} \sin \left(\frac{m \pi z}{H}\right) e^{i\left(k_{m} x-\omega_{0} t\right)}+\frac{\Psi_{n}(\tau)}{2} \sin \left(\frac{n \pi z}{H}\right) e^{i\left(k_{n} x-\omega_{0} t\right)} \\
& +\frac{\Psi_{p}(\tau)}{2} \sin \left(\frac{(n-m) \pi z}{H}\right) e^{i\left(\left(k_{p} x-2 \omega_{0} t\right)\right.}+\text { c.c. },
\end{aligned}
$$

where it is assumed that the same modal amplitudes exist at all $x$. It is noteworthy that the transient wave field in our experiments contains quite strong amplitude variations along $x$, too. In this subsection, however, we ignore these spatial variations to potentially capture the leading order temporal evolution of the amplitudes before they reach steady state.

Following a procedure that is very similar to how Eqs. (20) and (21) were derived, the amplitude evolution equations can now be shown to be

$$
\begin{gathered}
\frac{d \Psi_{j}}{d \tau}=i \beta_{j} \Psi_{q}^{*} \Psi_{p}, \quad \text { where }(j, q)=(m, n) \text { or }(n, m), \\
\frac{d \Psi_{p}}{d \tau}=i \beta_{p} \Psi_{m} \Psi_{n},
\end{gathered}
$$

where

$$
\beta_{j}=\alpha_{j} c_{g, j}=\alpha_{j}\left(\frac{\omega_{j}}{k_{j}}\right)\left(1-\frac{\omega_{j}^{2}}{N_{0}^{2}}\right) \text { for } j=m, n, \text { and } p .
$$

The coefficients $\alpha_{j}$ appeared earlier in the amplitude evolution Eqs. (20) and (21), and $c_{g, j}$ is the group speed of mode $j$ at frequency $\omega_{j}$.

We investigate the usefulness of Eqs. (29) and (30) in describing the temporal evolution of the observed transient wave field in the case- 1 resonant triad experiment, i.e., $(m, n, p)=$ $(3,4,1)$, forced at resonance. The temporal evolution (from very early times) of the primary and superharmonic wave amplitudes at two different $x$ locations are shown in Fig. 11. At $x=7.5$ $\mathrm{cm}$ [Fig. 11(a)], we observe that the primary wave amplitudes reach steady state by around $t=3 T_{0}-4 T_{0}$, by which point the superharmonic mode 1 has also started growing. The slope of initial growth of the superharmonic mode 1 is well captured by the theoretical slope of initial 
TABLE I. Theoretical [Eq. (34)] and experimentally observed spatial viscous decay rates $C_{j}\left(\mathrm{in}^{-1}\right.$ ) for mode 3 at $\omega_{0}$ (column 1), mode 4 at $\omega_{0}$ (column 2), and mode 1 at $2 \omega_{0}$ (column 3 ) from analytical expression (34) and individual mode experiments.

\begin{tabular}{lllc}
\hline \hline$C_{j}\left(\right.$ in $\left.^{-1}\right)$ & \multicolumn{1}{c}{$C_{3}$} & \multicolumn{1}{c}{$C_{4}$} & $C_{1}$ \\
\hline 2D [Eq. (34)] & 0.0238 & 0.0563 & 0.4608 \\
Individual mode experiments & 0.66 & 0.73 & - \\
\hline \hline
\end{tabular}

growth (black dashed-dotted line) based on Eqs. (29) and (30). A similar result holds at $x=21 \mathrm{~cm}$ [Fig. 11(b)], except that the slope of initial growth of the superharmonic mode 1 sustains for a longer duration. As a result, and as already discussed, the steady-state superharmonic mode- 1 amplitude is larger at $x=21 \mathrm{~cm}$ than at $x=7.5 \mathrm{~cm}$. In summary, Fig. 11 has shown that the slow temporal evolution theory is useful in describing the initial growth of the superharmonic mode- 1 wave at a fixed location, which subsequently saturates at its steady value. Once the wave field reaches steady state, the amplitude evolution equations (20) and (21) take over to quantitatively describe the spatial growth of the superharmonic mode 1 for distances of around one wavelength of the primary mode 3.

\section{B. Viscous effects}

As reported in Fig. 5, while the inviscid amplitude evolution equations (20) and (21) accurately capture the spatial growth of the superharmonic wave at small $x$, they evidently underestimate the spatial decay of the primary wave amplitudes. We approximately incorporate viscous effects in the amplitude evolution equations in the following manner:

$$
\begin{gathered}
\frac{d}{d x}\left(\epsilon \Psi_{j}\right)=i \alpha_{j} \epsilon \Psi_{q}^{*} \epsilon \Psi_{p}-C_{j} \epsilon \Psi_{j}, \text { where }(j, q)=(m, n) \text { or }(n, m), \\
\frac{d}{d x}\left(\epsilon \Psi_{p}\right)=i \alpha_{p} \epsilon \Psi_{m} \epsilon \Psi_{n}-C_{p} \epsilon \Psi_{p},
\end{gathered}
$$

where $C_{j}(j=m, n$, or $p)$ represents the spatial viscous decay rate for mode $j$ at its corresponding frequency. We recall that $\epsilon \Psi_{j}$ represents the modal amplitude in the physical wave field. In the absence of viscous effects, Eqs. (32) and (33) reduce to the inviscid amplitude evolution equations (20) and (21). In the absence of amplitude evolution due to triadic resonance, each modal amplitude evolves spatially as $\exp \left(-C_{j} x\right)$. The values of $C_{j}$ are directly measured from experiments where mode $j$ is forced in isolation at the corresponding frequency with a relatively small amplitude of $A_{j}=2 \mathrm{~mm}$. Before discussing the observed viscous decay rates, it is instructive to discuss their physical origins.

Based on weak viscous dissipation in linear, two-dimensional flows with a kinematic viscosity $v$, the spatial viscous decay rate of mode $j$ at frequency $\omega_{j}$ is [46]

$$
C_{j}^{2 D}=j^{3}\left(\frac{\pi}{H}\right)^{3} \frac{v}{2 N} \frac{1}{\left(1-\omega_{j}^{2} / N^{2}\right)^{5 / 2}} .
$$

The viscous decay rate in Eq. (34) for the modal amplitudes can be obtained as half of the product between the kinematic viscosity $v$, the inverse of the group speed $\partial \omega_{j} / \partial k_{j}$ (the speed with which energy, proportional to the square of the amplitude, propagates), and $\left[k_{j}^{2}+(j \pi / H)^{2}\right]$, which results from the Laplacian operator in the viscous term of the momentum equations. Equation (34) suggests that the decay rate goes as the cube of the mode number, hence resulting in larger viscous decay for higher modes. Furthermore, the closer $\omega_{j} / N$ is to unity, the larger the viscous decay is. For the three waves that form the case- 1 resonant triad, i.e., $(m, n, p)=(3,4,1)$ with $\omega_{0} / N=0.4841$, the corresponding values of $C_{j}^{2 D}$ are shown in Table I. Among the three waves, $C_{j}^{2 D}$ is largest for the 

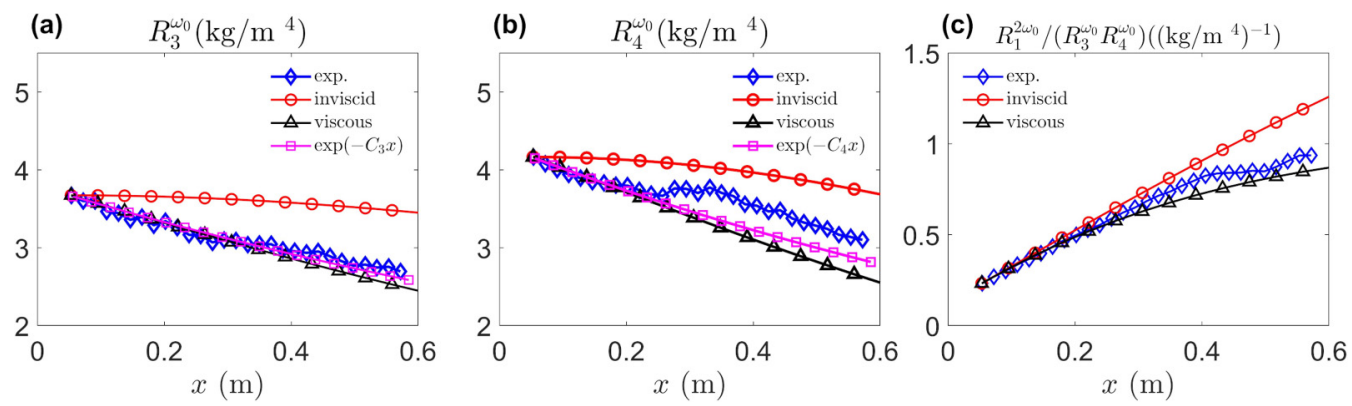

FIG. 12. Comparison between observations and inviscid/viscous theory at resonance for the case-1 resonant triad experiment presented in Fig. 5. (a) Spatial evolution of the experimental steady-state mode-3 amplitude ( $\diamond$ ). Predictions from the inviscid amplitude evolution Eqs. (20) and (21) and the viscous amplitude evolution Eqs. (32) and (33) are shown in red (o) and black $(\triangle)$, respectively. Shown in magenta $(\square)$ is the viscous decay of the primary mode 3 as observed in an individual mode- 3 experiment (observed decay rate $C_{3}$ is given in Table I). (b) Same as (a), but for the primary mode 4. (c) Spatial evolution of the experimental normalized steady-state superharmonic mode-1 amplitude $(\diamond)$. Predictions from the inviscid amplitude evolution Eqs. (20) and (21) and the viscous amplitude evolution Eqs. (32) and (33) are shown in red (०) and black $(\triangle)$, respectively. The normalization factor $R_{3}^{\omega_{0}} R_{4}^{\omega_{0}}$ is as measured at $x=5.5 \mathrm{~cm}$.

superharmonic mode 1 owing to its frequency being close to $N$. The measured viscous decay rates $C_{3}$ and $C_{4}$ for the individual primary modes are, however, an order of magnitude larger than what is estimated based on Eq. (34) (Table I). These significantly larger measured viscous decay rates can be attributed to the viscous effects acting at the side walls of the tank in the experiments [47], an effect that is not captured in a two-dimensional model such as that corresponding to Eq. (34). For the superharmonic mode 1, it was difficult to estimate its viscous decay rate from the individual mode experiment owing to the occurrence of parametric subharmonic instability [36]. As a result, the corresponding entry in Table I is left blank. For the viscous amplitude evolution equations, while we use the experimentally measured $C_{3}$ and $C_{4}$ as reported in the second row of Table I, $C_{p}=C_{p}^{2 D}$ is assumed for the superharmonic wave. Owing to the frequency of the superharmonic wave being close to $N_{0}$, it is reasonable to assume that the corresponding viscous decay is dominated by the $2 \mathrm{D}$ effect captured in Eq. (34).

Figure 12 shows a comparison between experimental observations and predictions from viscous amplitude evolution equations (32) and (33) for the case-1 resonant triad experiment at resonance, i.e., the same experiment as presented in Fig. 5. Figures 12(a) and 12(b) show the spatial evolution of the primary wave amplitudes, indicating a good quantitative agreement between observations and the viscous theory. Furthermore, the viscous damping estimated from the individual mode experiments [shown using $\square$ in Figs. 12(a) and 12(b)] also describe the observed spatial decay well. In summary, the observed decay is mainly due to viscous damping rather than energy transfer to the superharmonic wave. In contrast, the spatial growth of the superharmonic wave is described well by the inviscid theory, with the incorporation of viscous effects seemingly capturing the departure from linear growth from around $x=0.3 \mathrm{~m}$ [Fig. 12(c)].

\section{CONCLUSIONS}

In this paper, we have presented an experimental study of triadic resonance in internal wave modes when the primary wave field contains two waves of the triad. Using the internal wave generator to force two different modes simultaneously at the theoretically predicted resonant frequency, spontaneous excitation of a superharmonic wave of a mode number equal to the difference between the mode numbers of the primary waves was observed. The steady-state wave field was then shown to comprise a combination of spatially evolving internal wave modes that 
constitute the resonant triad. The spatial growth of the steady-state superharmonic wave at small distances from the wave generator is captured accurately by the inviscid amplitude evolution equations, based on the method of multiple scales. Indeed, the assumption of a large-scale spatial evolution of the modal amplitudes effectively describes the observed steady-state wave field, at least for small distances from the wave generator. Experiments at off-resonant frequencies suggest that superharmonic wave generation due to triadic resonance is non-negligible at least over a forcing frequency range (around the resonant frequency) of around 0.03 times the buoyancy frequency associated with the uniform stratification. Inviscid amplitude evolution equations based on a slow temporal evolution of the modal amplitudes were then shown to reasonably describe the transient evolution of the wave field towards steady state. Finally, viscous effects were shown to be the dominant factor in the spatial decay of steady-state primary wave amplitudes.

For a validation of the viscous theory over larger distances, it would be necessary to eliminate the effect of the right-to-left propagating waves reflected from the right end of the tank in the experiments. Also, a more rigorous treatment of viscous effects in deriving the amplitude evolution equations, i.e., by including viscous terms that capture decay due to $2 \mathrm{D}$ and side wall effects in the governing equations, may also improve the theoretical predictions of spatial evolution over larger distances. It would be interesting to perform experiments where primary waves of multiple resonant triads are simultaneously forced, a scenario that may occur in internal wave generation mechanisms like tide-topography interaction. The relative importance of superharmonic wave generation due to triadic resonance with respect to other mechanisms such as parametric subharmonic instability, interaction with the mean flow or topography, etc., remains to be explored for realistic oceanic regimes. Finally, incorporating the effects of nonuniform stratification [37,44,48], background rotation [44,49], and three dimensionality [50] would make our study more relevant for oceanic settings.

\section{ACKNOWLEDGMENTS}

The authors acknowledge funding from the Indian Ministry of Human Resource Development under the SPARC programme (Sanction Order No. SPARC/2018-2019/P1213/SL). D.V. and M.M. acknowledge funding and hospitality from ENS de Lyon during their stay at ENS de Lyon. This work was supported by Grant No. ANR-17-CE30-0003 (DisET) and by the LABEX iMUST (ANR-10-LABX-0064) of Université de Lyon, within the program Investissements d'Avenir (ANR11-IDEX-0007), operated by the French National Research Agency (ANR). It has been achieved thanks to the resources of PSMN from ENS de Lyon. M.M. also acknowledges funding from the Department of Science and Technology, India under the FIST Grant No. SR/FST/ET-II/2017/109.

[1] A. E. Gill, Atmosphere-Ocean Dynamics (Academic Press, Cambridge, 2016).

[2] W. Munk and C. Wunsch, Abyssal recipes II: Energetics of tidal and wind mixing, Deep-Sea Res. 45, 1977 (1998).

[3] M. J. Lighthill and J. Lighthill, Waves in Fluids (Cambridge University Press, Cambridge, 2001).

[4] R. H. J. Grimshaw and N. Smyth, Resonant flow of a stratified fluid over topography, J. Fluid Mech. 169, 429 (1986).

[5] B. Gayen and S. Sarkar, Turbulence During the Generation of Internal Tide on a Critical Slope, Phys. Rev. Lett. 104, 218502 (2010).

[6] R. M. Robinson, The effects of a vertical barrier on internal waves, Deep Sea Research and Oceanographic Abstracts 16, 421 (1969).

[7] O. Bühler and M. Holmes-Cerfon, Decay of an internal tide due to random topography in the ocean, J. Fluid Mech. 678, 271 (2011).

[8] J. T. Nault and B. R. Sutherland, Internal wave transmission in nonuniform flows, Phys. Fluids 19, 016601 (2007). 
[9] M. Mathur and T. Peacock, Internal Wave Interferometry, Phys. Rev. Lett. 104, 118501 (2010).

[10] K. Hasselmann, A criterion for nonlinear wave stability, J. Fluid Mech. 30, 737 (1967).

[11] A. Javam, J. Imberger, and S. W. Armfield, Numerical study of internal wave-wave interactions in a stratified fluid, J. Fluid Mech. 415, 65 (2000).

[12] K. R. Helfrich and W. K. Melville, Long nonlinear internal waves, Annu. Rev. Fluid Mech. 38, 395 (2006).

[13] T. Gerkema, Internal and interfacial tides: Beam scattering and local generation of solitary waves, J. Mar. Res. 59, 227 (2001).

[14] S. G. Llewellyn Smith and W. R. Young, Conversion of the barotropic tide, J. Phys. Oceanogr. 32, 1554 (2002).

[15] C. Garrett and E. Kunze, Internal tide generation in the deep ocean, Annu. Rev. Fluid Mech. 39, 57 (2007).

[16] M. H. Alford, J. A. MacKinnon, H. L. Simmons, and J. D. Nash, Near-inertial internal gravity waves in the ocean, Annu. Rev. Mar. Sci. 8, 95 (2016).

[17] C. Staquet and J. Sommeria, Internal gravity waves: From instabilities to turbulence, Annu. Rev. Fluid Mech. 34, 559 (2002).

[18] K. G. Lamb, Internal wave breaking and dissipation mechanisms on the continental slope/shelf, Annu. Rev. Fluid Mech. 46, 231 (2014).

[19] T. Dauxois, S. Joubaud, P. Odier, and A. Venaille, Instabilities of internal gravity wave beams, Annu. Rev. Fluid Mech. 50, 131 (2018).

[20] L. J. Sonmor and G. P. Klaassen, Toward a unified theory of gravity wave stability, J. Atmos. Sci. 54, 2655 (1997).

[21] S. J. Ghaemsaidi and M. Mathur, Three-dimensional small-scale instabilities of plane internal gravity waves, J. Fluid Mech. 863, 702 (2019).

[22] S. A. Thorpe, On wave interactions in a stratified fluid, J. Fluid Mech. 24, 737 (1966).

[23] C. R. Koudella and C. Staquet, Instability mechanisms of a two-dimensional progressive internal gravity wave, J. Fluid Mech. 548, 165 (2006).

[24] T. Gerkema, C. Staquet, and P. Bouruet-Aubertot, Decay of semi-diurnal internal-tide beams due to subharmonic resonance, Geophys. Res. Lett. 33, L08604 (2006).

[25] A. D. McEwan, Degeneration of resonantly-excited standing internal gravity waves, J. Fluid Mech. 50, 431 (1971).

[26] S. Martin, W. Simmons, and C. Wunsch, The excitation of resonant triads by single internal waves, J. Fluid Mech. 53, 17 (1972).

[27] A. D. McEwan, D. W. Mander, and R. K. Smith, Forced resonant second-order interaction between damped internal waves, J. Fluid Mech. 55, 589 (1972).

[28] D. Benielli and J. Sommeria, Excitation and breaking of internal gravity waves by parametric instability, J. Fluid Mech. 374, 117 (1998).

[29] A. Tabaei, T. R. Akylas, and K. G. Lamb, Nonlinear effects in reflecting and colliding internal wave beams, J. Fluid Mech. 526, 217 (2005).

[30] C.-H. Jiang and P. S. Marcus, Selection Rules for the Nonlinear Interaction of Internal Gravity Waves, Phys. Rev. Lett. 102, 124502 (2009).

[31] S. Smith and J. Crockett, Experiments on nonlinear harmonic wave generation from colliding internal wave beams, Exp. Thermal Fluid Sci. 54, 93 (2014).

[32] A. S. Korobov and K. G. Lamb, Interharmonics in internal gravity waves generated by tide-topography interaction, J. Fluid Mech. 611, 61 (2008).

[33] L. Gostiaux, H. Didelle, S. Mercier, and T. Dauxois, A novel internal waves generator, Exp. Fluids 42, 123 (2007).

[34] M. J. Mercier, D. Martinand, M. Mathur, L. Gostiaux, T. Peacock, and T. Dauxois, New wave generation, J. Fluid Mech. 657, 308 (2010).

[35] B. Bourget, T. Dauxois, S. Joubaud, and P. Odier, Experimental study of parametric subharmonic instability for internal plane waves, J. Fluid Mech. 723, 1 (2013).

[36] S. Joubaud, J. Munroe, P. Odier, and T. Dauxois, Experimental parametric subharmonic instability in stratified fluids, Phys. Fluids 24, 041703 (2012). 
[37] D. Varma, V. Chalamalla, and M. Mathur, Spontaneous superharmonic internal wave excitation by modal interactions in uniform and nonuniform stratifications, Dynamics of Atmospheres and Oceans 91, 101159 (2020).

[38] G. Oster, Density gradients, Sci. Am. 213, 70 (1965).

[39] Y. Dossmann, F. Pollet, P. Odier, and T. Dauxois, Mixing and formation of layers by internal wave forcing, J. Geophys. Res.: Oceans 122, 9906 (2017).

[40] P. Husseini, Interactions non-linéaires d'ondes internes de gravité et mélange en milieu stratifié, Ph.D thesis, Université de Lyon, 2019.

[41] S. B. Dalziel, G. O. Hughes, and B. R. Sutherland, Whole-field density measurements by synthetic schlieren, Exp. Fluids 28, 322 (2000).

[42] A. Fincham and G. Delerce, Advanced optimization of correlation imaging velocimetry algorithms, Exp. Fluids 29, S013 (2000).

[43] J. Sommeria, Uvmat toolbox for matlab, Technical Report No. LEGI/CNRS-UJF-INPG [http://coriolis. legi.grenoble-inp.fr].

[44] D. Varma and M. Mathur, Internal wave resonant triads in finite-depth non-uniform stratifications, J. Fluid Mech. 824, 286 (2017).

[45] A. H. Nayfeh, Perturbation Methods (John Wiley \& Sons, New York, 2008).

[46] P. Echeverri, M. R Flynn, K. B. Winters, and T. Peacock, Low-mode internal tide generation by topography: An experimental and numerical investigation, J. Fluid Mech. 636, 91 (2009).

[47] F. Beckebanze, C. Brouzet, I. N. Sibgatullin, and L. R. M. Maas, Damping of quasi-two-dimensional internal wave attractors by rigid-wall friction, J. Fluid Mech. 841, 614 (2018).

[48] B. R. Sutherland, Excitation of superharmonics by internal modes in non-uniformly stratified fluid, J. Fluid Mech. 793, 335 (2016).

[49] S. Wunsch, Harmonic generation by nonlinear self-interaction of a single internal wave mode, J. Fluid Mech. 828, 630 (2017).

[50] J. R. Munroe and K. G. Lamb, Topographic amplitude dependence of internal wave generation by tidal forcing over idealized three-dimensional topography, J. Geophys. Res. 110, C02001 (2005). 